\title{
Intergenerationenbeziehungen im bäuerlichen Milieu zu Beginn des 21. Jahrhunderts
}

\author{
Anja Eder · Sabine A. Haring-Mosbacher · Franz Höllinger
}

Angenommen: 12. Juli 2021 / Online publiziert: 5. August 2021

(C) Der/die Autor(en) 2021

Zusammenfassung Trotz des Strukturwandels in der Landwirtschaft hat sich die bäuerliche Lebensform der Mehrgenerationenfamilie in Österreich weitgehend erhalten. Allerdings stellt sich die Frage, wie sich diese Lebensform zu den tiefgreifenden Individualisierungstendenzen, die moderne Gesellschaften durchziehen, verhält. Anhand von 30 Interviews und einer österreichweiten Umfrage unter 269 Betriebsleiterinnen und -leitern untersucht der vorliegende Artikel, wie die Generationenbeziehungen von Alt und Jung wahrgenommen werden. Dazu greift der Beitrag auf soziologische Konzepte der Generationensolidarität, des Generationenkonflikts und der Ambivalenzen in den Generationenbeziehungen sowie auf die historische Familienforschung zurück. Die Analyse, die auch die Geschlechterverhältnisse mit einbezieht, zeigt eine Kontinuität der Familiensolidarität, zugleich aber einen erheblichen Wandel der Solidaritäts- und Konfliktmuster. Als charakteristisch für das bäuerliche Milieu zu Beginn des 21. Jahrhunderts erweisen sich der Wandel von der verpflichtenden zur freiwilligen Generationensolidarität, von traditionellen Abhängigkeitsverhältnissen zu einer autonomeren Lebensführung sowie mehr Privatsphäre und vom patriarchalen Gehorsam zur egalitären Familienkommunikation.

Schlüsselwörter Generationenbeziehung · Generationensolidarität ·

Generationenambivalenz · Generationenkonflikt · Patriarchaler Gehorsam

Anja Eder $(\bowtie) \cdot$ Sabine A. Haring-Mosbacher $\cdot$ Franz Höllinger

Soziologisches Seminar, Universität Graz, Universitätsstraße 15, 8010 Graz, Österreich

E-Mail: anja.eder@uni-graz.at

Sabine A. Haring-Mosbacher

E-Mail: sabine.haring@uni-graz.at

Franz Höllinger

E-Mail: franz.hoellinger@uni-graz.at 


\section{Intergenerational relationships in the farming milieu at the beginning of the $21^{\text {st }}$ century}

Abstract Despite the profound structural changes in the agricultural sector, farm families in Austria have widely maintained a way of life as multi-generational households. Still, the question arises as to how this form of life relates to the far-reaching individualization trends that pervade modern societies. Based on 30 interviews and an Austria-wide survey of 269 farm managers, this article examines how intergenerational relationships are perceived by the old and the young. To this end, the article draws on sociological concepts of generational solidarity, generational conflict and ambivalences in intergenerational relationships, as well as on historical family research. The analysis, which also focuses on gender relations, shows a general continuity of family solidarity, but also considerable changes in patterns of solidarity and conflict. The change from obligatory to voluntary generational solidarity, from traditional dependent relationships to a more autonomous lifestyle with increased privacy, and from patriarchal obedience to egalitarian family communication are characteristic of the peasant milieu at the beginning of the 21 st century.

Keywords Intergenerational relationships - Intergenerational solidarity · Generation conflict · Patriarchal obedience $\cdot$ Intergenerational ambivalences

\section{Les relations intergénérationnelles en milieu paysan au début du $21^{\mathrm{e}}$ siècle}

Résumé Malgré les transformations structurelles de l'agriculture, le mode de vie paysan de la famille multigénérationnelle s'est largement maintenu en Autriche. La question se pose cependant de savoir comment ce mode de vie s'articule aux profondes tendances à l'individualisation qui traversent les sociétés modernes. À partir de 30 entretiens et d'un sondage auprès de 269 chefs et de cheffes d'exploitation dans toute l'Autriche, le présent article étudie la façon dont les relations entre les générations sont perçues. Pour ce faire, cet article recourt aux concepts sociologiques de la solidarité, du conflit et de l'ambivalence dans les relations entre les générations ainsi qu'aux recherches historiques sur la famille. Cette analyse, qui tient également compte des rapports entre les sexes, met en évidence une continuité de la solidarité familiale mais aussi dans le même temps un changement net des motifs de solidarité et de conflit. Le milieu paysan du début du $21^{\mathrm{e}}$ siècle apparaît ainsi caractérisé par le passage de la solidarité obligatoire à la solidarité volontaire entre les générations, des rapports de dépendance traditionnels à un mode de vie plus autonome et plus respectueux de la sphère privée ainsi que de l'obéissance patriarcale à la communication familiale égalitaire.

Mots-clés relations intergénérationnelles · solidarité intergénérationnelle · ambivalence intergénérationnelle · conflit intergénérationnel · obéissance patriarcale 
„Es soll einmal ein anderer versuchen, 24 Stunden mit seinem Chef Arbeit und Freizeit gemeinsam zu verbringen. "

(Elfriede Sch., 49 Jahre, Leiterin eines bäuerlichen Familienbetriebs im Haupterwerb)

\section{Einleitung}

Bäuerliche Familien sind bis heute Familien mit Produktionsfunktion. Während es in anderen Berufsbranchen inzwischen nur mehr wenige Familienbetriebe gibt, findet die landwirtschaftliche Produktion in Österreich nach wie vor primär im Rahmen von Familienbetrieben statt, die von einer Generation an die nächste weitergegeben werden. Laut Statistik werden über $90 \%$ der Höfe in Österreich als Familienbetriebe geführt (Bundesministerium 2019, S. 62) und $82 \%$ aller land- und forstwirtschaftlichen Arbeiten von Familienarbeitskräften, darunter auch von Familienangehörigen der Hofeigentümer, geleistet (ebd., S. 66).

Obwohl der Strukturwandel in der Landwirtschaft vielfältige Veränderungsprozesse einleitete, blieb die bäuerliche Wirtschafts- und Lebensform in der Dreigenerationenfamilie weitgehend erhalten. Auch die innerfamiliäre Weitergabe von Höfen wird weiterhin der bevorzugte Weg bleiben - laut einer Umfrage der Bundesanstalt für Agrarwirtschaft ziehen lediglich $8 \%$ der Befragten eine außerfamiliäre Hofnachfolge in Betracht (Quendler et al. 2015, S. 7). Besonders charakteristisch ist dabei die innerfamiliäre Weitergabe des Hofes an den (ältesten) Sohn (Larcher und Vogel 2009, S. 68).

Damit ist die Mehrgenerationenfamilie, die in nächster Nähe zusammenlebt und -arbeitet, im bäuerlichen Milieu kein Auslaufmodell. Angesichts der zunehmenden Individualisierung, der wachsenden Bedeutung der Kernfamilie sowie der vermehrten Brüchigkeit von Familienbeziehungen (u.a. Beck und Beck-Gernsheim 1990, S. 69 ff; Beham-Rabanser et al. 2019, S. 181 ff.; Peuckert 2008) stellt sich gleichwohl die Frage, wie sich die bäuerliche Mehrgenerationenfamilie etwa mit den heutigen Idealvorstellungen der Autonomie des Ehepaars gegenüber der Elterngeneration vereinbaren lässt. Außerhalb des bäuerlichen Milieus manifestiert sich die zunehmende Individualisierung in einem markanten Anstieg von Einpersonenhaushalten, die im Jahr 2015 bereits mehr als ein Drittel aller österreichischen Haushalte ausmachten (Kaindl und Schipfer 2019, S. 13). Diese Entwicklung bildet auf den ersten Blick einen krassen Gegensatz zwischen dem bäuerlichen und dem außerbäuerlichen Milieu und erweckt den Anschein, als wären der familiäre Zusammenhalt und die Solidarität zwischen den Generationen in der Landwirtschaft wesentlich stärker ausgeprägt. Vor diesem Hintergrund untersucht der vorliegende Beitrag, wie Generationenbeziehungen am Bauernhof gestaltet und von den Angehörigen der jüngeren und der älteren Generation wahrgenommen werden. Dazu werden auf der Basis einer Interviewstudie Beziehungsnarrative über die Solidaritäts- und Konfliktmuster sowie die Ambivalenzen in den Generationenbeziehungen herausgearbeitet.

In soziologischen Zeitdiagnosen wird die Frage des Wandels von Generationsbeziehungen häufig im Lichte der Individualisierungsthese betrachtet und interpretiert. Im Mittelpunkt dieser Perspektive steht die Annahme eines historischen Wandels 
von traditionellen zu modernen und weiter zu postmodernen Familienbeziehungen (Lüscher 1993, S. 38 ff.) und der damit verbundenen ,Schwerpunktverlagerung vom Verwandtschaftsverband hin zur Kernfamilie und schließlich zum Individuum“ (Burkart 2008, S. 125) - wobei sich, so die Diagnose von Beck und Beck-Gernsheim in den 1990er-Jahren, die Brüchigkeit des traditionellen Familienmodells verstärken und auch bis dato ,stabile“ Bevölkerungsgruppen erreichen werde. Es komme zu einer zunehmenden räumlichen Distanz zwischen erwachsenen Kindern und ihrer Herkunftsfamilie, zur Betonung der jeweiligen Paarbeziehungen und zu einer immer stärkeren Fokussierung auf die Wünsche, Bedürfnisse und Interessen der einzelnen Individuen (vgl. u.a. Beck und Beck-Gernsheim 1990, 1994). Diese Entwicklung führe letztlich zu mehr ,Instabilität, mehr Wechsel, mehr Übergänge[n] und Zwischenformen im Lebenslauf“ (Beck-Gernsheim 1997, S. 77). BeckGernsheim thematisierte dabei stets die unterschiedlichen Individualisierungsvoraussetzungen männlicher und weiblicher Lebenslagen und die daraus resultierenden neuen Entscheidungsräume und Lebenschancen sowie Konflikte und Zwänge, die sich für Männer und Frauen in zum Teil unterschiedlicher Art und Weise ergeben bzw. mit welchen diese zu kämpfen haben. Davon ausgehend, legt der vorliegende Beitrag auch ein besonderes Augenmerk auf die Kontinuität und den Wandel geschlechtsspezifischer Generationenkonflikte.

Familiensoziologen und Familiensoziologinnen, die sich näher mit der Frage der Beziehungen zwischen den Generationen in der Gegenwartsgesellschaft auseinandergesetzt haben, wie Kurt Lüscher oder Vern L. Bengtson, bestreiten nicht, dass derartige Individualisierungsprozesse stattfinden. Sie betonen jedoch, dass Individualisierung nicht mit familiärer Entsolidarisierung gleichzusetzen ist (Lüscher 2000) und halten die Vorstellung der „Isolation der modernen Kleinfamilie“ für einen Mythos (Bengtson 2001). Charakteristisch für die heutigen Generationenbeziehungen sei vielmehr ein Wechselspiel von „Generationenkonflikt, Generationensolidarität und Generationenambivalenz" (Lüscher et al. 2014, S. 14; s. a. Höpflinger 1999, S. 22). Intergenerationenbeziehungen, so die Annahme, verlieren gegenüber früheren Epochen nicht an Bedeutung: Es ändert sich nur ihr Charakter und zum Teil gewinnen sie sogar an Relevanz.

Zum Wandel der Generationenbeziehungen im bäuerlichen Milieu liegen im deutschsprachigen Raum bislang nur wenige Studien vor, wie auch insgesamt der ländliche Raum und die Agrarsoziologie in der soziologischen Forschung nur eine untergeordnete Rolle spielen. Das Thema Landfamilie sei, so Bruno Hildenbrand (2005, S. 125), seit den 1970er-Jahren bis auf wenige Ausnahmen ,zunehmend aus der sozialwissenschaftlichen Diskussion“" verschwunden. Gleichzeitig fanden in der bisherigen Forschung zu Generationenbeziehungen auch die Geschlechterverhältnisse nur ein geringes Maß an Aufmerksamkeit (Helfferrich 2017). Diesen Forschungslücken widmet sich der vorliegende Beitrag. Indem wir die bäuerlichen Generationenbeziehungen zu gesamtgesellschaftlichen Entwicklungen einerseits sowie zu den historischen Ausgangsbedingungen in der Landwirtschaft anderseits in Beziehung setzen, versuchen wir, der Besonderheit des intergenerationalen Miteinanders auf den österreichischen Bauernhöfen auf die Spur zu kommen. Zugleich lassen sich von hier aus wieder allgemeinere Rückschlüsse auf das Verhältnis von Kontinuität und Wandel von Generationenbeziehungen im 21. Jahrhundert gewin- 
nen. Ist die bäuerliche Familie eine schlichte „Ausnahme“, die sich der Entwicklung zum postmodernen Familienleben entzieht, oder folgt sie dem gesamtgesellschaftlichen Entwicklungstrend?

Im folgenden Abschnitt werden zunächst die beiden theoretischen Ansätze präsentiert, die die Hintergrundfolie für unsere Analyse bilden: das Solidaritäts-Konflikt-Modell von Vern L. Bengtson et al. (2002) und das Konzept der Generationenambivalenz von Kurt Lüscher (2000). Da bei Lüscher das Spannungsfeld zwischen Solidarität und individuellen Ansprüchen im Mittelpunkt des Interesses steht, erweist sich sein Ansatz als besonders anschlussfähig für unsere Analyse. Abschnitt 3 gibt einen kurzen Überblick über den Forschungsstand zum Wandel der Intergenerationenbeziehungen am Bauernhof. In Abschnitt 4 wird die Methodik der von den Autorinnen und dem Autor dieses Beitrags durchgeführten Interview- und Fragebogenstudie vorgestellt. Abschnitt 5 präsentiert die zentralen Ergebnisse in drei Schritten: Zunächst wird auf die Kontinuität der intergenerationalen Familiensolidarität, dann auf den Wandel der Generationenbeziehungen im bäuerlichen Milieu eingegangen und schließlich der Zusammenhang zwischen Generationenkonflikten und Geschlechterbeziehungen erörtert. Ein abschließendes Fazit stellt die Ergebnisse nochmals zur Diskussion (Abschnitt 6).

\section{Generationensolidarität, Generationenkonflikt und Generationenambivalenz}

Entgegen der Annahme, dass die Individualisierung privater Lebensformen mit einer „Normalisierung der Brüchigkeit“ (Beck-Gernsheim 1997, S. 66) einhergehe und die Abnahme der Solidarität zwischen den Generationen sowie die Isolation, Vereinsamung und Abschiebung alter Menschen nach sich ziehen würde (z.B. Hoffmann-Nowotny 1995; Marshall et al. 1993), weisen eine Reihe von empirischen Untersuchungen darauf hin, dass (1.) Familiengenerationen auch in den westlichen Gegenwartsgesellschaften zumeist nicht weit voneinander entfernt wohnen (zumindest eines der erwachsenen Kinder lebt in der Nähe der Eltern) und somit sogenannte „multilokale Mehrgenerationen“ bilden (Burkart 2008, S. 205), dass (2.) die Generationen häufig telefonischen und/oder persönlichen Kontakt haben, dass sie sich (3.) gegenseitig unterstützen und (4.) in der Regel auch eng miteinander verbunden fühlen (Bertram 2000; Cooney und Dykstra 2013; Kohli und Szydlik 2000; Rosenbaum 2011). Angesichts zahlreicher derartiger Befunde halten Bengtson (2001) und Bertram (2000) die isolierte Kleinfamilie, im Sinne einer deutlichen Distanzierung zwischen den Generationen, für einen Mythos. In ähnlicher Weise glaubt auch Marbach (1994), dass der sogenannte Generationenvertrag bis heute nicht gefährdet ist. Prognosen für das fortschreitende 21. Jahrhundert gehen vielmehr von einer wachsenden Bedeutung intergenerationaler Beziehungen aus (Bengtson 2001, S. $4 \mathrm{f}$.). Dies hängt auch damit zusammen, dass sich die Lebensphase, in der drei Generationen gleichzeitig leben, durch die gestiegene Lebenserwartung deutlich verlängert hat (Cooney und Dykstra 2013, S. 356). Insbesondere werden die Großeltern immer wichtiger bei der Betreuung ihrer Enkelkinder, weil heute oftmals beide Elternteile einer Erwerbstätigkeit nachgehen und zudem die Zahl an Haushalten mit Alleiner- 
ziehenden zunimmt (Bengtson 2001, S. 4 ff.). Der Wichtigkeit der Großeltern ging ein tiefgreifender Wandel der Familie und der Geschlechterrollen seit den 1960erJahren voraus, der zumindest in westlichen Industriegesellschaften durch ein Abflachen der Hierarchien zwischen den Generationen, eine Pluralisierung familiärer Lebensformen und ein zunehmend egalitäres partnerschaftliches Miteinander in Eheund Lebensgemeinschaften gekennzeichnet ist (Burkart 2008). Trotz der Abnahme von Mehrgenerationenhaushalten gehört die Unterstützung bei der Kinderbetreuung seitens der Großeltern nach wie vor zu den ,unhinterfragten Selbstverständlichkeiten im Denken der Großelterngeneration“ (Brake und Büchner 2007, S. 200).

Beim Vergleich zwischen ländlichen und städtischen Familien konnte Hildenbrand allerdings feststellen, dass Verwandtschaftsnetzwerke in der Stadt stärker den „Charakter von Wahlverwandtschaften haben und in ihrer Bedeutung in dem Maße zurückgehen, in dem andere Optionen von Interessen sich eröffnen und konkrete Hilfeleistungen nicht im Netzwerk organisiert werden müssen, sondern gekauft werden können“ (Hildenbrand 2005, S. 127). Zudem verdeutlichen familiensoziologische Forschungen, dass intensive Kontakte zwischen den Generationen die Gefahr ernsthafter Konflikte in sich bergen, die in letzter Konsequenz zum Abbruch der Beziehungen führen können (Lüscher 2000, S. 147). Häufigere Kontakte zwischen den Generationen verstärken zudem die Ambivalenzen ${ }^{1}$ der Beziehungen (Lüscher 2000, S. 148) - es entsteht dann ein ,Spannungsfeld zwischen Unterstützung und Einmischung, Engagement und vornehmer Zurückhaltung“ (Brake und Büchner 2007, S. 200). Das Verhältnis zwischen den Generationen ist somit weniger geprägt durch eine zunehmende Brüchigkeit der Beziehungen als durch „Generationensolidarität, Generationenkonflikt und Generationenambivalenz" (Lüscher et al. 2014, S. 14). Mit dem Abflachen der Hierarchien zwischen den Generationen und den Geschlechtern hat sich insbesondere der Wert der Kommunikation verändert (Cooney und Dykstra 2013, S. 361).

In den 1990er-Jahren entwickelten Bengtson und Roberts (1991) ein multidimensionales Solidaritäts-Konflikt-Modell zur Beschreibung intergenerationaler Familienkohäsion. Solidarität manifestiert sich demnach durch häufige Interaktionen zwischen den Angehörigen der unterschiedlichen Generationen (assoziativ), durch emotionale Verbundenheit (affektiv), durch die Übereinstimmung von Einstellungen und Werten (konsensuell), durch den Austausch von Ressourcen und Hilfeleistungen (funktional) sowie durch die Verpflichtung, den Kontakt zu den Familienangehörigen zu pflegen und diese zu unterstützen (normativ). Höhere Solidaritätswerte auf einer Dimension können, müssen aber nicht mit höheren Werten auf anderen Dimensionen einhergehen (Bengtson und Roberts 1991, S. 857 f.). Solidarität bedeutet hierbei keineswegs das Fehlen von Konflikten und Ambivalenzen (Szydlik 2008, S. 101; s. a. Szydlik 2000, S. 41). Um dies zu verdeutlichen, ordnen Bengtson et al. den einzelnen Solidaritätsformen Ambivalenzen zu, wie etwa der affektiven Solidarität die Ambivalenz zwischen Intimität und Distanz oder der normativen Solidarität die Ambivalenz zwischen Familialismus und Individualismus (Bengtson et al. 2002).

\footnotetext{
1 Einen Überblick zum Thema „Generationenambivalenz“ geben u.a. Pillemer und Müller-Johnson (2007).
} 
Abb. 1 Modell der Generationenambivalenz nach Lüscher (Quelle: Lüscher 2000, S. 154; grafisch leicht angepasst)

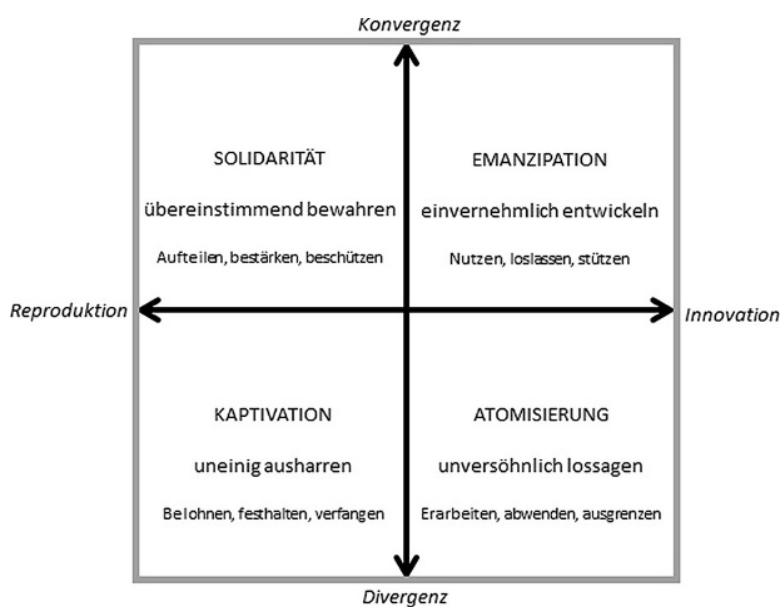

Noch stärker als Bengtson et al. betont Kurt Lüscher die Ambivalenz in den heutigen Intergenerationenbeziehungen und schließt hier in weitem Sinne an namhafte Einschätzungen u.a. von Bauman (1992) an, der Ambivalenz als das Merkmal der Moderne beschreibt. Lüscher meint, dass vor allem ,ideelle und normative Abhängigkeiten“ zwischen den Generationen ,ein wesentlicher Anstoß für Ambivalenzen“ sind (Lüscher 2000, S. 140). Er entwickelt eine Typologie der Generationenambivalenz, die einen ,Zusammenhang zwischen Deutungsmustern, Handlungsmaximen und Beziehungslogiken“ herstellt (2000, S. 154; siehe Abbildung 1). Generationenbeziehungen stehen demnach im Spannungsfeld zwischen dem Bedürfnis nach Kontinuität und dem Bedürfnis nach Veränderung (Reproduktion versus Innovation) einerseits sowie dem Bedürfnis nach Nähe und dem Wunsch nach Distanz (Konvergenz versus Divergenz) andererseits (siehe Abbildung 1). Aus der Kombination dieser beiden Bedürfnisachsen ergeben sich vier Typen der Generationenbeziehungen: Solidarität (übereinstimmend bewahren), Emanzipation (einvernehmlich neue Beziehungsmuster entwickeln), Kaptivation (trotz Distanzbedürfnis ausharren) und Atomisierung (sich unversöhnlich voneinander lossagen) (Lüscher 2000, S. 155 f.).

In verschiedenen Studien wurde zudem seit längerem untersucht, ob und inwiefern die Generationenbeziehungen von Jung und Alt unterschiedlich wahrgenommen werden. Mit der ,intergenerational-stake-Hypothese“ gingen Bengtson und Kuypers (1971) davon aus, dass Eltern weniger Konflikte wahrnehmen und über eine größere emotionale Nähe berichten als ihre Kinder. Erklärt wurde dies damit, dass die Elterngeneration eher an „,der Kontinuität von Werten im Generationenzusammenhang und an einer engen Beziehung zu ihren Kindern interessiert" (Kopp und Steinbach 2009, S. 2) ist und deshalb positive Aspekte der Beziehung stärker hervorhebt als die Generation der Kinder. Dagegen zeigen aktuellere Analysen, wie beispielsweise jene von Kopp und Steinbach (2009, S. 10), dass die Unterschiede in der Wahrnehmung von Alt und Jung deutlich geringer sind, als dies im Rahmen der ,intergenerationalstake-Hypothese" bislang angenommen wurde.

Bevor vor dem Hintergrund der Modelle von Bengtson und Roberts resp. Lüscher die gegenwärtigen Generationenbeziehungen österreichischer Bäuerinnen und 
Bauern näher analysiert und, unter besonderer Berücksichtigung der Geschlechterverhältnisse, deren Kontinuitäten und Diskontinuitäten im zeitlichen Verlauf nachgezeichnet werden, gilt es, kurz auf die zentralen Befunde der historischen Familienforschung zum bäuerlichen Milieu im Speziellen einzugehen. Erst durch diese historische Perspektivierung wird es möglich, die Persistenz und den Wandel intergenerationaler Solidaritäts- und Konfliktformen innerhalb des bäuerlichen Milieus $\mathrm{zu}$ verstehen, in gesamtgesellschaftliche Entwicklungen einzuordnen und auch zu der Veränderung der Geschlechterbeziehungen in Bezug zu setzen.

\section{Generationenbeziehungen im bäuerlichen Milieu}

Die charakteristische Form des bäuerlichen Familienbetriebs in Österreich war der Bauernhof, auf dem das bäuerliche Ehepaar und seine Kinder, die Altbauern sowie das Gesinde zusammenlebten und arbeiteten (Bruckmüller 2002). Die sozialen Beziehungen zwischen den Hofbewohnern und Hofbewohnerinnen waren den wirtschaftlichen Interessen untergeordnet und folgten einer patriarchalen Hierarchie (Rosenbaum 1982, S. 116; s. a. Mitterauer und Sieder 1991). Am traditionellen Bauernhof gab es keine klare räumliche Trennung zwischen Wohn- und Arbeitsbereich; Privat-, Berufs- und öffentliches Leben flossen ineinander (Bruckmüller et al. 2002, S. 415; für die Forstwirtschaft siehe auch Westermayer 2006, S. 222 f.). Die Altbauern hatten in der Regel einen eigenen Wohnbereich; sie nutzten diesen aber meist nur zum Schlafen. Tagsüber arbeiteten sie am Hof mit, die arbeitsfreien Zeiten verbrachte man in der Bauernstube.

Seit dem 19. Jahrhundert und zunächst außerhalb des bäuerlichen Milieus lässt sich ein Wandel von der Lebensform des „Ganzen Hauses“ hin zur „,bürgerlichen“ Kernfamilie feststellen, in der Wohnung und Arbeitsstätte räumlich getrennt sind (Burkart 2008; Hermann 2008). Die bürgerliche Familie zieht sich in einen Privatraum zurück, der zunehmend durch Intimität und Emotionalität gekennzeichnet ist (Beck und Beck-Gernsheim 1990, S. 69 ff.). Mit der Trennung von Familien- und Arbeitsleben kommt es zu einer Polarisierung der Geschlechterrollen. Dem Mann wird die Rolle des Ernährers zugeschrieben, die Frau wird aus der Arbeitswelt ausgeschlossen, auf das Haus verwiesen und mit der Erziehung der Kinder als ihrer „ureigensten“ Aufgabe betraut (Meyer 2008, S. 332).

Seit den 1960er-Jahren lässt sich in westlichen Industriegesellschaften wiederum ein tiefgreifender Wandel der Familie beobachten: Die sogenannte postmoderne Familie ist gekennzeichnet durch ein Abflachen der Hierarchien zwischen den Generationen, eine Pluralisierung familiärer Lebensformen, ein egalitäres partnerschaftliches Miteinander in Ehe- und Lebensgemeinschaften, aber auch durch eine zunehmende Brüchigkeit von Beziehungen.

Für bäuerliche Familien sind in der unmittelbaren Nachkriegszeit (nach dem Ende des Zweiten Weltkriegs) jedoch zunächst die Mechanisierung der Landwirtschaft und der Anstieg der Löhne für landwirtschaftliche Arbeitskräfte besonders bedeutsam. Denn diese führten letztendlich zur Auflösung der Gesindegesellschaft. Die vielfältigen Arbeiten am Bauernhof sind jedoch nach wie vor wesentlich leichter zu bewältigen, wenn sich mehrere Personen daran beteiligen, sodass die Betriebe 
seit damals stärker auf die Mithilfe der Altbauern angewiesen waren als früher. Andererseits hat sich durch den Bedeutungsverlust des Ausgedinges und durch die Einführung einer staatlichen Pensionsversicherung die Abhängigkeit zwischen den Generationen gelockert (Goldberg 2003, S. 112). Diese abnehmende Abhängigkeit zwischen den Generationen geht jedoch nicht mit einem Bedeutungsverlust der bäuerlichen Familienbetriebe einher. Vielmehr lässt sich, wie der Historiker Ernst Langthaler (2012) herausarbeitet, eine „resilience through hybridity“ (S. 401) beobachten, die es Familienbetrieben ermöglicht, trotz des tiefgreifenden Wandels der Nachkriegszeit in großer Zahl zu überleben.

Im deutschsprachigen Raum gibt es nur relativ wenige Studien zum Wandel der Generationenbeziehungen im bäuerlichen Milieu (zu den bedeutenderen Beiträgen zählen: Fliege 1998; Geserick et al. 2006; Goldberg 2003; Hildenbrand et al. 1992; Pongratz 1990, 1991). Ein zentrales Thema der vorhandenen Untersuchungen ist die Frage, wie sich die traditionelle Form der bäuerlichen Familie mit den ökonomischen Anforderungen und den soziokulturellen Gegebenheiten der modernen Gesellschaft vereinbaren lässt. Mehrere Autoren und Autorinnen vertreten die Ansicht, dass Tradition und Moderne für bäuerliche Familien keinen antagonistischen Gegensatz darstellen. So stellt etwa Hans Pongratz fest: „Anscheinend wehren bäuerliche Familien moderne Anforderungen [...] nicht mit traditionellen Vorstellungen $\mathrm{ab}$, sondern verbinden traditionelle und moderne Elemente in einer den bäuerlichen Lebensbedingungen angepassten Weise" (Pongratz 1990, S. 20). Charles Hennon und Bruno Hildenbrand (2005) vertreten sogar die These, dass die traditionelle Wirtschaftsform des bäuerlichen Familienbetriebs nur dann überlebensfähig ist und aufrechterhalten werden kann, wenn sowohl der Modus der landwirtschaftlichen Produktion als auch die bäuerliche Familie moderne Züge annehmen.

Aufgrund der unsicheren Zukunftsperspektiven landwirtschaftlicher Betriebe und des zunehmenden Anspruchs auf freie Berufswahl ist es heute nicht mehr selbstverständlich, dass Bauernkinder den Hof ihrer Eltern übernehmen (Goldberg 2003; Rossier 2005). Dies hat zu einer Veränderung der Machtbalance zugunsten der jüngeren Generation geführt. Die Eltern müssen sich mehr als früher darum bemühen, attraktive Bedingungen für die Hofnachfolge zu schaffen. Auch der Anstieg des Bildungsniveaus und die Verkürzung der Halbwertszeit des landwirtschaftlichen Wissens begünstigen die Position der jüngeren Generation. Andererseits kann es dadurch vermehrt zu intergenerationellen Konflikten kommen (Brake und Büchner 2007, S. 204).

Da außerdem der Kreis potenzieller Heiratspartner und Heiratspartnerinnen aus dem bäuerlichen Milieu kleiner wird, kommt es immer häufiger vor, dass sich Hofnachfolger und Hofnachfolgerinnen für Partner und Partnerinnen entscheiden, die keinen landwirtschaftlichen Hintergrund haben. Hildenbrand et al. (1992) konnten in ihren Untersuchungen feststellen, dass Jungbäuerinnen, die nicht aus dem bäuerlichen Milieu stammen, weniger bereit sind, sich an die etablierten Normen des Zusammenlebens im bäuerlichen Mehrgenerationenhaushalt anzupassen, und mehr darauf achten, die Autonomie der Kernfamilie gegenüber der älteren Generation am Hof zu sichern als Bäuerinnen, die selbst von einem Bauernhof stammen. Auch vermehrt vorkommende Nebenerwerbstätigkeiten dürften dazu beitragen, dass berufliche Anforderungen und Normen der nicht-bäuerlichen Arbeitswelt auf die bäuer- 
liche Familie einwirken und das Verhältnis zwischen den Generationen beeinflussen (Rossier 2005).

\section{Fragestellungen, Daten und Analysemethoden}

Vor dem Hintergrund des skizzierten Forschungsstands sollen folgende Fragestellungen anhand des von uns erhobenen Datenmaterials beantwortet werden:

1. Wie wird die für den bäuerlichen Familienbetrieb charakteristische und für die Aufrechterhaltung des Betriebs notwendige Lebensform der Dreigenerationenfamilie von der älteren und von der jüngeren Generation wahrgenommen?

2. Kam es nach Auffassung der Befragten in den letzten Jahrzehnten zu einem Wandel der Beziehungen zwischen den Generationen? Welche Solidaritäts- und Konfliktmuster sowie Ambivalenzen in den Generationsbeziehungen zeigen sich in den Narrativen der Befragten?

3. Welche typischen Beziehungs- und Konfliktmuster lassen sich im Verhältnis zwischen Alt- und Jungbauer einerseits sowie Altbäuerin und (angeheirateter) Jungbäuerin andererseits feststellen? Inwieweit haben sich diese geschlechtsspezifischen Beziehungsmuster in den letzten Jahrzehnten geändert?

Grundlage für unsere empirischen Analysen in diesem Beitrag sind Daten, die im Rahmen des FWF-Forschungsprojekts „Perspektiven für bäuerliche Familienbetriebe in Österreich“ im Zeitraum 2014 bis 2016 erhoben wurden. ${ }^{2}$ Die Studie beruht auf einem Mixed-Methods-Design und umfasst eine österreichweite Fragebogen-Befragung von 269 Leiterinnen und Leitern von landwirtschaftlichen Familienbetrieben sowie 30 qualitative Interviews mit Bauern und Bäuerinnen in der Steiermark. ${ }^{3}$ Zentrale Themenbereiche in beiden Teilen der Studie sind die Arbeitsteilung und das Zusammenleben der Angehörigen des bäuerlichen Familienverbands, die Produktionsstruktur und Arbeitsweise sowie die Frage nach den Zukunftsperspektiven des landwirtschaftlichen Betriebs. In den qualitativen Interviews wurde zudem untersucht, wie sich die ökonomische Situation des Betriebs und die sozialen Beziehungen zwischen den Familienangehörigen in den letzten Jahrzehnten entwickelt und verändert haben. Die Auswahl der Befragten erfolgte bei beiden Erhebungsteilen nach einem Quotenplan: beim Fragebogen nach Alter und Geschlecht der Befrag-

\footnotetext{
2 Zum Projektteam gehörten Anja Eder, Eva Maria Griesbacher, Franz Höllinger und Sabine A. Haring. Am Projekt maßgeblich beteiligt waren auch Eduard Ulreich, Leopold Kirner und Liane Kaipel von der Hochschule für Agrar- und Umweltpädagogik Wien. Die wichtigsten Ergebnisse des Projekts wurden im Buch Bäuerliche Lebenswelten in Österreich am Beginn des 21. Jahrhunderts (Höllinger et al. 2017) veröffentlicht.

3 Die Interviews wurden aus Zeit- und Kostengründen nur in einem Bundesland geführt. Die Steiermark deckt im Unterschied zu anderen österreichischen Bundesländern alle Typen der bäuerlichen Produktion in Österreich ab und war aus diesem Grunde als geografischer Raum für unsere qualitativen Interviews besonders geeignet. Auch gehen wir davon aus, dass die Generationenbeziehungen am Bauernhof in den verschiedenen Regionen Österreichs im Wesentlichen ähnlich sind, auch wenn es aufgrund regional unterschiedlicher Rahmenbedingungen (z.B. historisch tradierte Erbschaftsregelungen) gewisse Unterschiede geben könnte.
} 
ten sowie nach der Erwerbsform des Betriebs (Haupt- vs. Nebenerwerb); bei den Interviews zusätzlich nach Produktionsschwerpunkten. ${ }^{4}$

Alle Interviews fanden am Bauernhof statt und dauerten ein bis drei Stunden. Auf etwa zwei Drittel der untersuchten Höfe lebten zum Zeitpunkt der Befragung drei Generationen. Die Interviews wurden in der Regel mit dem Betriebsleiterehepaar geführt. Bei einem Teil der Interviews wurde nur eine Person (der Bauer oder die Bäuerin) befragt; häufig jedoch wurde das Ehepaar gemeinsam interviewt; manchmal beteiligten sich auch andere Familienangehörige am Gespräch, die während des Interviews in der Bauernstube anwesend waren. Zusätzlich wurden drei Fallstudien durchgeführt, bei denen neben dem Betriebsleiterehepaar auch die Altbauern sowie der potenzielle Hofnachfolger bzw. die potenzielle Hofnachfolgerin in eigenen Interviews befragt wurden. ${ }^{5}$ Neben Fragen nach Betriebsstruktur, Arbeitsweise und -teilung, familiären Strukturen und Praktiken, sozialen und ökonomischen Netzwerken sowie Zukunftsvorstellungen wurden die Interviewten auch gebeten, die Geschichte des Hofes zu erzählen, ihre Kindheitserinnerungen zu verbalisieren sowie gegenwärtige Erfahrungen mit früheren in Beziehung zu setzen.

Die Auswertung der qualitativen Interviews erfolgte mittels der strukturierenden Inhaltsanalyse nach Udo Kuckartz (2012). Mit dem Ziel einer grundlegenden Strukturierung des Interviewmaterials wurden in einem ersten Schritt der Analyse übergeordnete Kategorien längeren Interviewpassagen zugeordnet. Die deduktiv gewonnenen Kategorien flossen bereits in die Erstellung der Interviewleitfäden ein und umfassten (1.) das Generationen-Verhältnis, (2.) die Familiendynamiken, (3.) die Rollen und Aufgaben der Familienmitglieder, (4.) die Wohnsituation, (5.) den Zusammenhalt und Konflikte sowie (6.) den Ruhestand. Im Anschluss an die grundlegende Strukturierung des Materials anhand dieser sechs vorläufigen Grobkategorien wurden im Projektteam in einem schrittweisen und kleinteiligen Prozess, anhand des ständigen Vergleichs von Interviewpassagen und Fällen, Kategorien aus dem Interviewmaterial herausgearbeitet. Im Zuge dieser induktiven Kategorienbildung konnte der subjektive Sinn der zentralen Narrative der Bauern und Bäuerinnen rekonstruiert und historisch eingeordnet werden. ${ }^{6}$ Beim sinnrekonstruktiven Vorgehen wurde verstärkt mit in-vivo Codes gearbeitet, um nicht vorschnell eine zu große

\footnotetext{
${ }^{4}$ Der Vergleich unserer Studie mit den Ergebnissen der österreichischen Agrarstatistik (laut Grüner Bericht 2016) zeigt, dass der Anteil nicht-konventioneller Betriebe in unserer Stichprobe höher ist als in der Gesamtheit der landwirtschaftlichen Betriebe: $29 \%$ der Befragten der Fragebogenerhebung betreiben biologische Landwirtschaft, in der Gesamtheit der österreichischen landwirtschaftlichen Betriebe liegt dieser Anteil bei 17\%; $20 \%$ der Betriebe unserer Stichprobe, aber nur 11\% aller österreichischen Bauernhöfe leisten Maschinenring-Dienste; der Anteil der Betriebe, die Urlaub auf dem Bauernhof anbieten, ist in unserer Stichprobe etwa doppelt so hoch (13\%) wie in der Gesamtpopulation (6\%). Diese Verzerrungen sind darauf zurückzuführen, dass die Leiterinnen und Leiter solcher Betriebe leichter zugänglich sind und eher bereit waren, an der Befragung teilzunehmen (vgl. Höllinger et al. 2017, S. 13 ff.).

5 Die Suche nach Dreigenerationenfamilien, die bereit waren, sich auf dieses Setting einzulassen, gestaltete sich schwierig. Ein Grund für die mangelnde Bereitschaft war vermutlich die Sorge, dass innerfamiliäre und vor allem intergenerationale Konflikte zur Sprache kommen könnten, die ein schlechtes Licht auf eine der Generationen und/oder einzelne Familienmitglieder werfen könnten.

6 Im Sinne der Interkoderreliabilität wurde die induktive Kategorienbildung der Autorinnen und des Autors dieses Beitrags kontinuierlich in Interpretationssitzungen diskutiert, miteinander verglichen und überarbeitet. Erste Interpretationssitzungen fanden zudem bereits nach den ersten Interviews statt. Die Erkenntnisse dieser ersten Interpretationssitzungen sind auch in die Auswahl weiterer Fälle eingeflossen.
} 
Distanz zu den Narrativen herzustellen. Die rekonstruierten Narrative ließen sich am Ende des Forschungsprozesses vor allem drei Tendenzen zuordnen: dem Wandel von der verpflichtenden zur freiwilligen Generationensolidarität, dem Wandel von traditionellen Abhängigkeitsverhältnissen zu einer autonomeren Lebensführung und dem Wandel vom patriarchalen Gehorsam zur egalitären Familienkommunikation. Die Untersuchung des Wandels der Generationenbeziehungen stützte sich dabei zum einen auf den Vergleich zwischen der Situationsdarstellung in der jüngeren und in der älteren Generation, zum anderen auf die Darstellung der Veränderung der Generationenbeziehungen (seit der Zeit ihrer Kindheit) durch die Befragten selbst. Bzgl. aller drei Tendenzen erwiesen sich die Geschlechterverhältnisse als besonders relevant für das Verständnis von Kontinuität und Wandel der Generationenbeziehungen auf den österreichischen Bauernhöfen; sie werden daher von uns nicht als Querschnittsmaterie behandelt, sondern in einem gesonderten Abschnitt dargestellt.

\section{Empirische Ergebnisse}

Im Folgenden werden die Ergebnisse entlang der drei zuvor skizzierten zentralen Forschungsfragen dargestellt.

\subsection{Kontinuität der intergenerationalen Familiensolidarität}

Entgegen der pauschalen Annahme einer ,Schwerpunktverlagerung vom Verwandtschaftsverband hin zur Kernfamilie und schließlich zum Individuum“ (Burkart 2008, S. 125) zeigt sich in den von uns geführten Interviews eine deutliche Kontinuität der intergenerationalen Unterstützung und Solidarität im bäuerlichen Milieu. Diese betrifft im Besonderen die intergenerationale Unterstützung bei der Kinderbetreuung, bei der Hof- und Hausarbeit sowie bei der Altenpflege. Angesichts der ökonomischen Notwendigkeit der intergenerationalen Unterstützungsleistungen kann hier zunächst der Eindruck entstehen, dass es sich vorwiegend um funktionale Tauschbeziehungen (Bengtson und Roberts 1991) handelt, nämlich um den Austausch nicht-monetärer Ressourcen zwischen den Generationen. Aus dieser Perspektive leistet die ältere Generation Unterstützung bei der Kinderbetreuung und den Hof- und Hausarbeiten, da sie sich erhofft, dass der Hof weitergeführt wird und die junge Generation später für ihre Pflege aufkommen wird (siehe klassische Theorien zu sozialen Tauschbeziehungen wie z.B. bei Homans 1961). Obwohl funktionaler Solidarität zwischen den Generationen eine erhebliche Bedeutung im bäuerlichen Milieu zukommt, spielen aber auch emotionale Verbundenheit in der Großfamilie und damit affektive Solidarität eine Rolle sowie tradierte Rollenvorstellungen und -erwartungen, die Bengtson und Roberts mit dem Begriff der normativen Solidarität fassen.

Aus unseren Interviews geht hervor, dass sich die Altbauern und -bäuerinnen geradezu selbstverständlich und mit viel Freude an der Kinderbetreuung beteiligen. Angesichts des großen Arbeitsaufwands auf den Betrieben, dem steigenden Trend zum Nebenerwerb und der weit verbreiteten Ablehnung von Fremdbetreuung ist die Mithilfe der Altbauern bei der Kinderbetreuung für den erfolgreichen Fortbestand des Hofs ökonomisch notwendig. Elisabeth Beck-Gernsheim spricht hier von 
„Hintergrundpersonen“, welche die Elternschaft wesentlich erleichtern. So berichten viele Befragte, dass die Vereinbarkeit der Betreuung von Kindern und der landwirtschaftlichen Tätigkeit ohne Unterstützung der (Schwieger-)Eltern gar nicht möglich wäre. Doch nicht nur schätzt die Generation der Betriebsleiterinnen die Unterstützung der Elterngeneration bei der Kinderbetreuung, auch die Großeltern freuen sich über den engen Kontakt zu den Enkelkindern. Der 65-jährige Hans E. betont die Bedeutung der großelterlichen Unterstützung bei der Kinderbetreuung, wie er sie als Kind selbst erfahren hat und nun seinerseits weitergibt.

„Und wenn es gut geht zwischen Jung und Alt, sehe ich nur, dass es ein großer Vorteil ist [...] für die nachkommenden Generationen und Kinder. Wie gerne kommen unsere Enkelkinder heute her. Und umgekehrt ist es früher auch so gewesen, wie viel meine Eltern auf unsere Kinder geschaut haben, auch wenn eines noch in den Windeln war. Und das weiß ich sehr zu schätzen, dass die das damals so gemacht haben. Ich hab' es immer sehr zu schätzen gewusst, dass sie mir helfen und wir dadurch Zeit gehabt haben für die Freizeitgestaltung. [...] Ich denke mir nur, wenn ich heute mit den Enkelkindern spiele, so viel Zeit hatte ich nie mit den eigenen. Und das sehe ich schon positiv. Weil wenn ich früher heimgekommen bin, sind sie meistens schon schlafen gegangen gewesen. Im Winter haben wir schon viel gespielt, aber noch intensiver kann ich mich heute mit den Enkelkindern beschäftigen. Dadurch sage ich, Großfamilie ist immer noch angebracht." (Hans E., 65)

Die großelterliche Unterstützung bei der Kinderbetreuung ist also kein neues Phänomen, sondern vielmehr Bestandteil eines informellen Generationenvertrags, der sowohl für die Alt- als auch für die Jungbauern unhinterfragt gilt. Für Hans E. jedenfalls ist „Großfamilie [...] immer noch angebracht“, vor allem dann, wenn es ein gutes Einvernehmen zwischen den Generationen gibt. Jenseits bloß funktionaler Solidarität zeigt sich, dass die Familienform der Großfamilie, die Verbundenheit zwischen Jung und Alt insbesondere dann als positiv erachtet wird, wenn die Generationenbeziehungen vor allem vom Bedürfnis nach Konvergenz und Reproduktion (Lüscher 2000, S. 155) geprägt sind. Dass sowohl die Alt- als auch die Jungbauern und -bäuerinnen Fremdbetreuung weitgehend ablehnen, macht deutlich, dass neben funktionaler und affektiver Solidarität auch gemeinsame Werte im Hinblick auf die Kinderbetreuung geteilt werden - mit Bengtson et al. kann dies als eine konsensuelle Solidaritätsbeziehung zwischen den Generationen charakterisiert werden. So erklärt sich auch das große Engagement der Großeltern, deren Betreuung der Kinder oftmals das Ausmaß einer Teilzeitbeschäftigung annimmt. Dass die Eltern des 65jährigen Hans E. die Enkelkinder beaufsichtigt haben, um dem Paar auch Raum für Freizeitgestaltung zu ermöglichen, ist in dieser Generation allerdings eher unüblich, insofern zumeist die Arbeit am Betrieb im Vordergrund stand.

Letztlich ist es insbesondere die Selbstverständlichkeit der intergenerationalen Unterstützung, die das bäuerliche Milieu von anderen Milieus unterscheidet. Europaweite Studien zeigen, dass außerhalb des bäuerlichen Milieus weniger als die Hälfte der Großeltern bei der Betreuung der Enkelkinder unterstützt (Cooney und Dykstra 2013, S. 358). Und selbst wo das der Fall ist, haben die Großeltern häufig nur abends oder an den Wochenenden Zeit für ihre Enkelkinder (die häufig auch 
gar nicht in derselben Stadt oder Gemeinde leben) und Termine müssen erst mühsam ausgehandelt werden. Dagegen ist die Gelegenheitsstruktur am Bauernhof - mit Szydlik (2000) die ,strukturelle Solidarität“ - eine wichtige Voraussetzung für die intergenerationale Solidarität in Sachen Kinderbetreuung.

Eine weitere Kontinuität intergenerationaler Familiensolidarität betrifft die Mithilfe der Altbauern bei den Hofarbeiten und Arbeiten im Haushalt. Wie bei der Kinderbetreuung lässt sich in den Erzählungen eine Grundhaltung der Selbstverständlichkeit erkennen, die auf der ökonomischen Notwendigkeit der intergenerationalen Unterstützung aufbaut, aber nicht dabei stehen bleibt. Nicht nur die Mithilfe auf dem Feld oder im Stall wird geschätzt, sondern auch die Unterstützung im Haushalt, sei es bei der Wäsche, beim Geschirrspülen oder beim Kochen. Die Generationen können sich aufeinander verlassen, ihre wechselseitige Unterstützung in familiären und betrieblichen Belangen wird als selbstverständlich vorausgesetzt.

Auch für die Altbauern steht es außer Frage, nach der Hofübergabe weiter in der Landwirtschaft mitzuhelfen, solange es gesundheitlich möglich ist. So ist es für die 54-jährige Aloisia H. selbstverständlich, ihren Sohn bei der Rindermast und beim Verkauf im Hofladen zu unterstützen. Dabei bedarf die Zusammenarbeit der Absprache und Abstimmung mit den Familienmitgliedern - Arbeits- und Freizeit müssen klar geregelt sein. Eine Verpflichtung zur Mithilfe lehnen aber auch die Altbauern ab:

„Ich denk' mir, so wie es in einer Landwirtschaft ausschaut, so wie es bei uns in der Direktvermarktung [direkter Verkauf landwirtschaftlicher Produkte an die Endverbraucher, Anm. d. Verf.] ausschaut und das einfach so bleibt, wird einfach das sein, dass wir wahrscheinlich immer mithelfen werden, solange es die Gesundheit erlaubt. Ja. Und [Pause]. Ja. Und es wird sicher akzeptiert werden, wenn ich dann sag', [... ] okay, du, heute den Tag bin ich einfach nicht da. Da müssen sie einfach schauen, wie das geht oder? Da bin ich dann einen halben Tag da oder so hin und her.“ (Aloisia H., 54)

Insbesondere wird von den Bauern und Bäuerinnen das gemeinsame oder abwechselnde Kochen füreinander sehr geschätzt, bei dem sich die Familie trifft und gegenseitig austauschen kann. Viele Befragte wechseln sich - selbst wenn die Familie in getrennten Haushalten lebt - beim Kochen ab, da es allen Beteiligten Zeit und Aufwand spart. Auch das ist eine Besonderheit des bäuerlichen gegenüber vielen anderen Milieus, wo generationenübergreifendes Kochen füreinander bestenfalls ein außeralltägliches Ereignis darstellt.

Schließlich beinhaltet der Generationenvertrag im bäuerlichen Milieu auch, dass die jüngere Generation für die Organisation der Pflege der Altbauern Sorge trägt und diesen ein Leben am Hof bis zu ihrem Lebensende ermöglicht. Die Pflege wird im bäuerlichen Milieu seit jeher in den Übergabeverträgen geregelt; und zwar zu einem Zeitpunkt, an dem die Altbauern in den meisten Fällen noch rüstig sind und die Pflegebedürftigkeit noch in weiter Ferne liegt. Die Pflichten der Jungbauern hinsichtlich der Pflege haben sich im Lauf der Zeit stark verändert (siehe Abschnitt 5.2), die Übernahme der Verantwortung für deren Organisation am bäuerlichen Betrieb ist aber auch heute noch eine unhinterfragte Norm. Obwohl es Probleme bei der Pflege der Schwiegermutter gab, weil sich diese nicht pflegen lassen und keine Hilfe anneh- 
men wollte, antwortet die 56-jährige Josefa K. auf die Frage, ob sie die Pflege der Schwiegermutter auch heute noch einmal übernehmen würde, dass sie sich ,,ja fast dazu verpflichtet" gefühlt hätte. In den von uns geführten Interviews wird deutlich, dass die eigenhändige innerfamiliäre Pflege der Altbauern heute in der Regel nicht mehr gefordert wird. Die Inanspruchnahme von Altersheimen ist aber nach wie vor unüblicher als in anderen Teilen der Gesellschaft, und der Wunsch der alten Menschen, am eigenen Hof gepflegt zu werden, ist für Jung und Alt ein gleichermaßen legitimer.

Bis hierhin hat unsere Untersuchung in den Bereichen der Kinderbetreuung, der Hof- und Hausarbeit sowie der Altenpflege eine erhebliche Kontinuität der Generationensolidarität im bäuerlichen Milieu gezeigt. Obwohl die intergenerationale Unterstützung in diesen drei Bereichen durchaus (auch) als funktional beschrieben werden kann, spielt sowohl affektive Solidarität - die emotionale Verbundenheit in der Mehrgenerationenfamilie - als auch normative Solidarität - das Teilen gemeinsamer Werte und Ansichten - eine entscheidende Rolle. Die Abhängigkeitsverhältnisse zwischen den Generationen lassen sich demnach nicht auf funktionale Aspekte reduzieren, sie sind ebenso ,ideell und normativ“ (Lüscher 2000, S. 140).

\subsection{Wandel der Generationenbeziehungen}

Die grundsätzliche Bereitschaft, den solidarischen Zusammenhalt in der bäuerlichen Dreigenerationenfamilie aufrechtzuerhalten, bedeutet aber keineswegs, dass sich die Beziehungen zwischen den Generationen am Bauernhof nicht verändert haben und nach wie vor ändern. In unseren Interviews lässt sich ein deutlicher Wandel in den Generationenbeziehungen feststellen, der darauf zurückzuführen ist, dass die jüngere Generation am Bauernhof die gesamtgesellschaftliche Entwicklung hin zu partnerschaftlichen Beziehungsidealen und zu einem höheren Anspruch auf Intimität und Emotionalität in der Kernfamilie mitvollzieht. Die Neugestaltung der Beziehung zwischen Alt- und Jungbauern betrifft insbesondere drei Aspekte, die jeweils in mehreren Interviews angesprochen und reflektiert wurden: den Wandel (1) von verpflichtender zu freiwilliger Generationensolidarität, (2) von traditionellen Abhängigkeitsverhältnissen zu einer autonomeren Lebensführung sowie mehr Privatsphäre und (3) vom patriarchalen Gehorsam zur egalitären Familienkommunikation.

\subsubsection{Von der verpflichtenden zur freiwilligen Generationensolidarität}

Im Zuge der Enthierarchisierung der Generationenbeziehungen werden auch die Aushandlungsprozesse im bäuerlichen Milieu zusehends auf Augenhöhe geführt. Hinzu kommt, dass die Übernahme der elterlichen Landwirtschaft heute in vielen Fällen nicht mehr als Privileg erachtet wird, sondern es im Gegenteil immer schwieriger wird, innerfamiliäre Hofnachfolger und Hofnachfolgerinnen zu finden (Groier 2004). ${ }^{7}$ Diese Entwicklung verschärfte sich noch durch die veränderten Rahmenbedingungen, v.a. durch die zunehmend neoliberale Marktentwicklung seit den 1990er-

\footnotetext{
7 Zwischen 1951 und 2010 wurden pro Jahr durchschnittlich 4400 Betriebe geschlossen (Bundesministerium für Land- und Forstwirtschaft, Umwelt und Wasserwirtschaft 2014).
} 
Jahren, die großen wirtschaftlichen Druck auf die bäuerlichen Betriebe ausübt (Kirner 2017). Aus früheren Studien ist zudem bekannt, dass die Wahrscheinlichkeit zur Hofübernahme sinkt, wenn potenzielle Hoferben und Hoferbinnen in einer außerlandwirtschaftlichen Tätigkeit Kompetenzen erwerben und dabei neue und vor allem städtische Lebensweisen kennenlernen (Stiglbauer und Weiss 2000, S. 12). Starke intergenerationale Konflikte erhöhen außerdem das Risiko, dass die Landwirtschaft von der Nachfolgegeneration nicht mehr übernommen wird (Kaplan et al. 2009).

Das zunehmende Miteinander der verschiedenen Generationen auf Augenhöhe führt auch dazu, dass althergebrachte Selbstverständlichkeiten in der Hofnachfolge, aber auch in der Kinder- und Altenbetreuung hinterfragt und diskutiert werden, wobei sich ein Wandel von Verpflichtung zu Freiwilligkeit besonders deutlich im Bereich der Altenpflege erkennen lässt. Wie im vorigen Abschnitt gezeigt wurde, besteht zwar die Verpflichtung zur Organisation der Pflege fort, sie geht jedoch nicht mehr mit der Erwartung einher, dass die Pflege innerfamiliär - d.h. im Regelfall durch die Schwiegertöchter - übernommen wird. Diese Erwartung, die selbst dann galt, wenn die Schwiegertöchter gleichzeitig eigene Kinder zu betreuen hatten, verliert im Zuge der Enthierarchisierung der Generationenbeziehungen, der Egalisierung der Geschlechterrollen und letztlich auch durch den steigenden ökonomischen Druck, der die zeitlichen Ressourcen für die Pflege der Altbauern einschränkt, an Bedeutung. Verpflichteten sich früher die Hoferben und -erbinnen noch, die Pflege der Eltern persönlich zu übernehmen, empfehlen Expertinnen und Experten von Landwirtschaftskammern heute, vertraglich zu vereinbaren, dass sich die junge Generation lediglich um die Organisation der Pflege kümmert (z.B. Kalkus 2016, S. 36). ${ }^{8}$

Die genauen Vereinbarungen zur Pflege sind auch im bäuerlichen Milieu zu einem wichtigen Aushandlungsgegenstand geworden, der eine offene Kommunikation zwischen den Generationen notwendig macht. Der 39-jährige Haupterwerbsbauer Michael F. berichtet, dass seine Mutter von ihm verlange, bis zu einer gewissen Pflegestufe selbst für sie zu sorgen; sobald sie diese überschreitet, „,darf“ er externe Hilfe zur Pflege in Anspruch nehmen. Er bezeichnet diese Forderung als ,großzügig“ von der Mutter, die überdies auch die Geschwister des Hofnachfolgers in die Pflicht der Finanzierung der elterlichen Pflege nimmt.

„Nur, wir als Landwirtschaft, wir werden, von uns wird einmal von Haus aus verlangt, unsere Alten - Entschuldigung, wenn ich das so bezeichne - oder unsere Eltern bis zum Tode hin zu pflegen. Dass sich das aber mit einer modernen Landwirtschaft dann leider nicht mehr so vereinbaren lässt, will keiner sehen. Da muss ich wieder sagen, haben meine Eltern oder vielmehr meine Mutter sehr großzügig bei der Übergabe, hat sie schon geschrieben, ab einer Pflege-, ich weiß es jetzt nicht mehr genau, aber ab einer gewissen Pflegestufe bin ich von der häuslichen Pflege, da bei uns da, entbunden. Weil sie, die Mama, das gesehen hat, das ist dann für uns als Betriebsnachfolger nicht mehr machbar. Und dann darf ich sie dann abgeben, ah, und dann sind auch meine Geschwis-

\footnotetext{
8 Durch die Einführung der Bauernpension und des staatlichen Pflegegeldes haben sich auch die finanziellen Verpflichtungen der jüngeren Generation gegenüber den Altbauern verringert.
} 
ter mitverantwortlich. Auch finanziell mitverantwortlich, dass das funktioniert. Aber bis zu einer gewissen Stufe hab' ich das sozusagen mitgekriegt, mitvererbt gekriegt (lachend), wenn man das so sagen will, und das Ausgedinge und blablabla, was alles dazugehört." (Michael F., 39)

Michael F. sieht die Verpflichtung zur Pflege durchaus kritisch. Er ist sich der Tatsache bewusst, dass sie speziell im bäuerlichen Milieu lange Gültigkeit beanspruchte und nach wie vor teilweise beansprucht, obwohl sie gerade mit der landwirtschaftlichen Tätigkeit schwer vereinbar ist. Er spricht sich daher hinsichtlich der Frage der Altenpflege für einen Normwandel im bäuerlichen Milieu aus.

Unsere Interviews machen den Wandel von der Verpflichtung zur Freiwilligkeit greifbar, verdeutlichen aber zugleich, dass dieser Wandel kein linearer Prozess ist, der im bäuerlichen Milieu als abgeschlossen betrachtet werden kann. Auch wenn die Freiwilligkeit beispielsweise bei der Hofübernahme oder Pflege zugenommen hat, zeichnen sich die Generationenbeziehungen in vielerlei Hinsicht durch die Ambivalenz von Freiwilligkeit und Verpflichtung aus. Studien aus anderen Milieus belegen, dass ein schlechter Gesundheitszustand der Elterngeneration und vor allem die Notwendigkeit von Pflegeleistungen die Ambivalenz zwischen den Generationen steigern (Cooney und Dykstra 2013, S. $361 \mathrm{f}$.). Die ältere Generation ist in vielen Fällen bestrebt, an Traditionen festzuhalten, um den Betrieb und die familiale Ordnung aufrecht zu halten, während sich die Nachfolgegeneration von Traditionen zusehends löst und sich von der Elterngeneration emanzipiert.

\subsubsection{Von traditionellen Abhängigkeitsverhältnissen zu einer autonomeren Lebensführung sowie mehr Privatsphäre}

Der Wandel von der Verpflichtung zur Freiwilligkeit ist eng verbunden mit der Veränderung der Abhängigkeitsverhältnisse zwischen den Generationen, insofern den Hofnachfolgern und -nachfolgerinnen immer mehr Autonomie zugestanden wird. Wie in Abschnitt 5.1 gezeigt wurde, sind diese jedoch gleichzeitig auch abhängig von der Unterstützung der Altbauern, womit sich die Ambivalenz der Generationenbeziehung tendenziell verstärkt.

Sich tolerant gegenüber der anderen Generation zu zeigen, ihre Lebens- und Arbeitsweise zu respektieren und sich trotz einer fortwährenden gegenseitigen Abhängigkeit ausreichend Autonomie zuzugestehen, wird im bäuerlichen Milieu insbesondere durch die räumliche Nähe erschwert. Während Wohn- und Arbeitsstätte in anderen Milieus bereits im 19. Jahrhundert getrennt wurden, bestehen im bäuerlichen Milieu gemeinsame Wohneinheiten zumindest teilweise fort. Auf den österreichischen Bauernhöfen gibt es ganz unterschiedliche Haushalts- und Wohnarrangements. Es finden sich beispielsweise getrennte Stockwerke, eine teilweise Trennung von Stockwerken, mit getrennten Hauseingängen oder ohne diese, mit einer eigenen Küche für jede Generation oder einer gemeinsamen Küche, Zimmer der Enkelkinder in verschiedenen Stockwerken und dergleichen mehr. Die Wohnarrangements verändern sich in der Regel im Lauf der Zeit, die Wohnräume werden zum Beispiel getrennt, wenn Kinder geboren werden und die neue Kernfamilie einen eigenen privaten Raum für sich beansprucht. 
Sind die Wohnräume der Generationen nicht voneinander getrennt, trägt dies immer wieder zu Konflikten bei, auch zu Spannungen innerhalb der Partnerschaft, da keine Rückzugsorte vorhanden sind. Durch die räumliche Nähe der Altbauern, die nach wie vor am Hof oder zumindest in unmittelbarer Nachbarschaft am Betrieb in Auszugshäusern leben, kommt es häufig zu Situationen, in denen sich die Altbauern in die Belange der Nachfolgegeneration einmischen. Die Abgrenzung wird noch zusätzlich erschwert durch die gemeinsame Hof- und Hausarbeit und durch die Unterstützung bei der Kinderbetreuung.

Unsere Studie zeigt, dass Freiraum und Privatsphäre als besonders wichtige Voraussetzungen für ein gutes soziales Klima auf den österreichischen Höfen erachtet werden. Die 47-jährige Auguste A. beschreibt die Situation des gemeinsamen Wohnraums rückblickend als sehr belastend, vor allem in jener Zeit, als ihre Kinder noch klein und betreuungsintensiv waren. Besonders negativ blieb ihr in Erinnerung, dass ihre Privat- und Intimsphäre beim Stillen der Kinder nicht nur von den Schwiegereltern, sondern auch von familienfremden Personen, die am Hof ein- und ausgingen, missachtet wurde. Auch die schlechte Vereinbarkeit der Kinderbetreuung mit den landwirtschaftlichen Tätigkeiten war für Auguste A. rückblickend sehr herausfordernd:

„Wir haben ja noch unten im Stammhaus gewohnt, noch lange. Wir sind erst zwölf Jahre heroben [also im eigenen Haus, Anm. d. Verf.]. Die Kinder sind unten auf die Welt gekommen. Zwei Hunde, drei Männer und ein Haufen anderer Leute, und dann sollst du stillen und ständig waren Handwerker da, immer Handwerker. Und die haben ja auch da gegessen. Und dann haben wir rigolen [den Boden umgraben - Anm. d. Verf.] müssen im Weingarten, da gesetzt, dort gesetzt, es war eh ständig etwas. [...] Ich bin halt gerade noch die Generation. Die jetzigen Jungen, wenn man so schaut, die sind schon gescheiter, die machen das schon anders.“" (Auguste A., 47)

Freiraum und Privatsphäre gewähren die notwendige Autonomie und werden in den Interviews auch von den jüngeren Befragten immer wieder als besonders wichtige Voraussetzungen für ein gutes Zusammenleben in der Dreigenerationenfamilie genannt - so auch vom 39-jährigen Tobias St., der mit seiner Frau, seinen vier Kindern und seinen Eltern einen Hof betreibt.

„Ja. Und da hat es dadurch sicher ein bisschen Reibereien gegeben, muss man sagen. Auch dadurch, dass man in der Küche ständig zusammenlebt und ja. Der Rückzugspunkt ist halt nachher auch relativ klein geworden. Dann sind die Kinder gekommen. Dann hast du ein Schlafzimmer und ein Wohnzimmer und ein Kind noch dazu. Und das ist einfach, wenn du irgendetwas ausreden willst und du kannst das nur im Schlafzimmer machen, das trägt es mit der Zeit nicht. Du brauchst einfach deinen Freiraum. So ist einfach die Zeit und das ist auch wichtig, sage ich. Und ich denke mir, eine gute Familie oder eine gute Zusammenarbeit in der Familie kann nur funktionieren, [...] wenn man sich nicht einfach ständig auf den Wecker geht.“ (Tobias St., 39)

Auf dem gemeinsamen Hof von Tobias (39) und Katharina (38) St. war es Tobias' Mutter, die den Schritt zum Auszug gemacht hat, auf anderen landwirtschaftlichen 


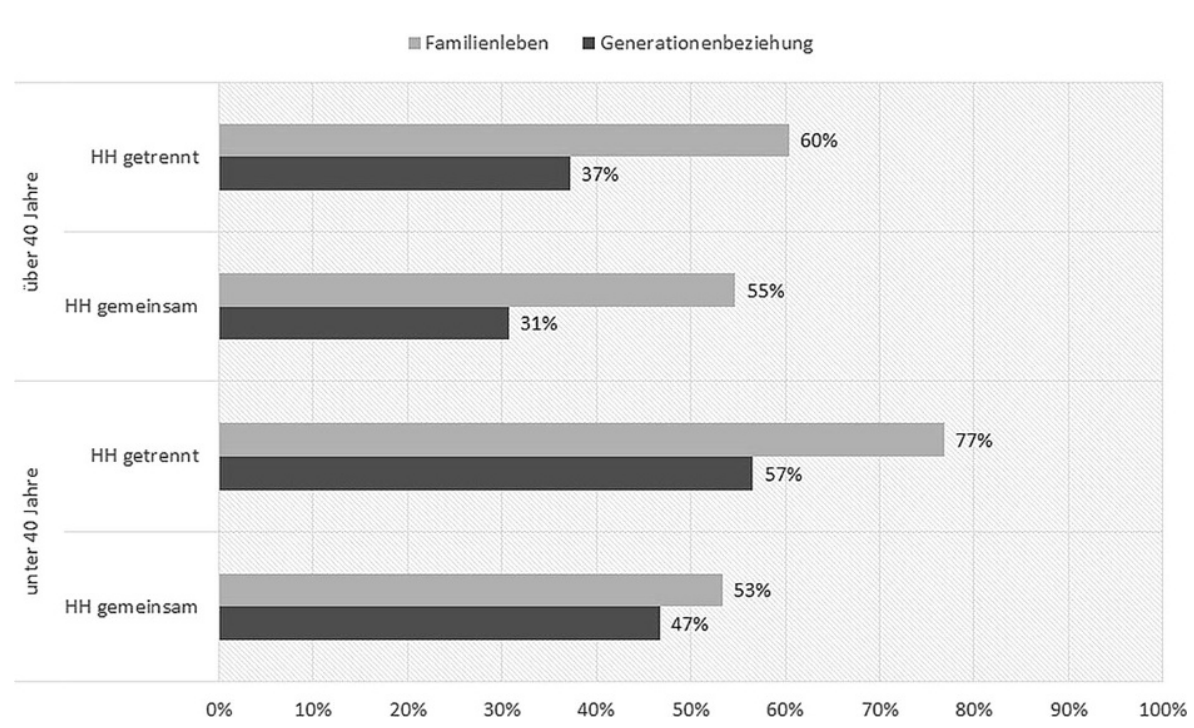

Abb. 2 Zufriedenheit mit Familienleben und Generationenbeziehungen unter jüngeren und älteren Befragten in getrennten und gemeinsamen Haushalten (\% zufrieden) (Quelle: Eder und Haring 2017, S. 263)

Familienbetrieben wird die Trennung der Haushalte häufig von der jüngeren Generation forciert. Auch unsere Fragebogenerhebung liefert Hinweise darauf, dass sich eine Trennung der Haushalte von Jung- und Altbauern positiv auf das Familienklima auswirkt. Bei klar getrennten Haushalten ist die Zufriedenheit mit dem Zusammenleben der Generationen deutlich höher als bei (teilweise) gemeinsamen Haushalten. Besonders zufrieden sind jüngere Bauern und Bäuerinnen, die auf Höfen leben, wo die Haushalte getrennt sind (siehe Abbildung 2; Eder und Haring 2017, S. 263).

Getrennte Wohnräume sind zwar keine Garantie dafür, dass sich die Generationen am Hof gegenseitig respektieren, sie schaffen jedoch Distanz, ermöglichen wichtige Rückzugsorte und fördern so die Autonomie der Generationen. Dies erleichtert zugleich den Umgang mit unterschiedlichen Anschauungen und Alltagspraxen in puncto Partnerschaft, Ehe, Kindererziehung oder Betriebsführung - und kann dadurch die emotionale Verbundenheit der Generationen sogar stärken. Was Tartler (1961) als ,,innere Nähe durch äußere Distanz“ beschreibt und Brauer (2002) in seiner Kontrastierung städtischer mit ländlichen Generationenbeziehungen aufgreift, lässt sich demnach - wenn auch unter anderen Wohn- und Arbeitsbedingungen - auch im bäuerlichen Milieu beobachten. Der Wandel hin zu mehr Autonomie bedeutet jedenfalls nicht, dass Generationenbeziehungen auf nurmehr funktionale Tauschbeziehungen reduziert werden - auch wenn der Fortbestand des Betriebs ein übergeordnetes Anliegen der Beteiligten bleibt. Deutlich wird vielmehr, dass das Streben nach Autonomie mit allen Arten von Solidaritätsverpflichtungen - affektiven, konsensuellen und normativen - in Einklang zu bringen ist. 


\subsubsection{Vom patriarchalen Gehorsam zur egalitären Familienkommunikation}

Der allmähliche Wandel vom patriarchalen Gehorsam zur egalitären Familienkommunikation stellt Alt- und Jungbauern vor die Herausforderung, neuartige Aushandlungsprozesse bewältigen zu müssen. Aushandlungsprozesse werden vor allem dort nötig, wo die junge Generation individuelle Bedürfnisse artikuliert, Veränderungen einfordert und dabei mit Traditionen hinsichtlich Partnerschaft, Kindererziehung und Betriebsführung bricht. Latente Meinungsverschiedenheiten zwischen den Generationen werden dadurch offenkundig, alte Konflikte treten an die Oberfläche und neue Konflikte werden wahrscheinlicher. Diese Konflikte werden begünstigt durch (1.) eine unzureichende und asymmetrische Kommunikation und Interaktion, (2.) durch unterschiedliche Lebensstile der Generationen, (3.) durch verschiedene Ansichten hinsichtlich der Kindererziehung, (4.) durch unterschiedliche Weltanschauungen, (5.) durch divergierende Auffassungen von Arbeit und Arbeitsleistung sowie (6.) durch verschiedene Praxen in der Haushaltsführung (Clarke et al. 1999, S. 263 ff.). Die Generationen stehen also vor der Herausforderung, ausreichend Konvergenz herzustellen, um weiterhin gemeinsam am Hof arbeiten und leben zu können, insofern diese eine Voraussetzung für Emanzipation, gegenseitiges Loslassen und einvernehmliche Weiterentwicklung darstellt (Lüscher 2000, S. 156).

Unsere Interviews zeigen, dass Konflikte zwischen den Generationen beispielsweise dann entstehen, wenn die Leitungsfunktion der nachkommenden Generation nach der Hofübernahme seitens der Altbauern nicht respektiert wird. Der Rollenwechsel vom Betriebsleiter bzw. der Betriebsleiterin zum Altbauern bzw. zur Altbäuerin wird in diesen Fällen als Kontrollverlust empfunden. Auch den Jungbauern fällt es oftmals schwer, mit dem nötigen Selbstbewusstsein gegenüber den Altbauern aufzutreten und etwa neue Wege in der Betriebsführung einzuschlagen.

In vielen Fällen müssen sich die Jungbauern und Jungbäuerinnen erst gegenüber den Altbauern behaupten. So musste der 39-jährige Haupterwerbsbauer Tobias St. seinen Vater auch nach der Hofübernahme von seinen Ideen zur Weiterentwicklung des landwirtschaftlichen Betriebs überzeugen.

„Ja. Also solange dir die Wirtschaft nicht gehört, musst du dich fügen, ist so. Was nachher mit der Übernahme dazukommt, du willst einfach deine Ideen richtig verwirklichen. Ich muss sagen, vom Vater her, also es war... Es war auch vorher so, dass die Mutter herinnen war und der Vater hat draußen die Arbeit gemacht. Und ich bin dadurch auch immer mit ihm beisammen oder beisammen gewesen. Er hat mich, muss ich sagen, nie richtig - nein, nie - gehindert an meinen Ideen. [...] Also ich habe ihm das immer plausibel erklären müssen, wie und was ich mir da vorstelle. Und dann hat er mich auch gelassen, muss ich sagen.“ (Tobias St., 39)

Die ältere Generation ringt in vielen Fällen mit der Enthierarchisierung der Generationenbeziehungen, der zunehmenden Gleichberechtigung der Geschlechter in heutigen Partnerschaften oder der Abkehr von autoritären Erziehungsmodellen. Die 47-jährige Auguste A. berichtet, dass der Schwiegervater noch einer Generation von Männern angehörte, ,die sich selber noch bedienen lassen haben im eigenen Haus. Der ist dann gekommen, hat sich reingesetzt und erwartet, dass er bedient wird.“ 
Auguste A. hat versucht, sich von diesen Erwartungen zu emanzipieren, und sich damit gegen die tradierten Vorstellungen aufgelehnt.

Da die unterschiedlichen Generationen am Hof miteinander arbeiten und sich gegenseitig unterstützen, ist es von besonderer Bedeutung, offen und ehrlich miteinander reden zu können. Für die 45-jährige Birgit P. gilt das insbesondere dann, wenn die Arbeitslast groß ist und die Gemüter angespannt sind. Sie betreibt gemeinsam mit ihrem Mann eine Rinderzucht und Milchwirtschaft im Haupterwerb und bietet zusätzlich Urlaub am Bauernhof an. Hierbei fallen viele Arbeiten an, die gemeinsam mit den Schwiegereltern bewältigt werden. Eine offene Kommunikation ist für Birgit P. unabdingbar, um den Familienfrieden dauerhaft aufrechtzuerhalten. Die egalitäre Kommunikation zwischen dem Betriebsleiterpaar und der (Schwieger-)Elterngeneration trägt aus ihrer Sicht entscheidend zur produktiven Zusammenarbeit bei. Spannungen und Tiefen gehören dabei ihrer Auffassung nach zum Zusammenleben der Generationen am Hof dazu; diese gäbe es auch im außerbäuerlichen Milieu.

„Es gibt überall, wie in jeder Familie, Höhen und Tiefen. Und es gibt Zeiten, wo man super gut miteinander zusammenarbeitet. Und dann gibt es Zeiten, wo Spannungen sind. Was wichtig ist dabei, dass man miteinander reden kann. Und auch, dass man dann redet miteinander. Also nicht nur, dass man es kann, sondern, dass man es auch dann tut. Was wir alle vier - also jetzt die Eltern und wir - immer wieder versuchen und was uns relativ gut gelingt, glaube ich, wo man aber schon merkt, wenn Spitzenarbeitszeiten sind. Also während des Sommers, wenn wirklich jeder angespannt ist und jeder bis aufs Letzte arbeiten muss. Dann wird es oft auch schwierig." (Birgit P., 45)

Auch der 36-jährige Stefan J. thematisiert die Bedeutung partnerschaftlicher Familienkommunikation und sieht darin einen Wandel gegenüber dem früher dominierenden patriarchalen Gehorsam. „Es war früher, war das halt, gut, der Junge muss still sein und der Alte hat das Recht. Und inzwischen ist das halt anders, nicht. Und für mich hat ja Respekt damit zu tun, dass das schon in beide Richtungen geht." Der frühere einseitige Respekt, der ausschließlich von der jungen gegenüber der älteren Generation eingefordert wurde, gehört seiner Ansicht nach der Vergangenheit an.

\subsection{Kontinuität und Wandel geschlechtsspezifischer Generationenkonflikte}

In Österreich dominiert nach wie vor die patrilineare Vererbungslinie (Larcher und Vogel 2009, S. 68). Frauen übernehmen den Hof nur dann, wenn keine männliche Geschwisterkonkurrenz vorhanden ist, wenn die Betriebe klein und wenig ertragreich sind und in einigen Fällen auch nur pro forma, weil der Mann einer außerlandwirtschaftlichen Tätigkeit nachgeht (auch aus Sozialversicherungsgründen); zudem sind sie seltener Alleineigentümerinnen (Larcher und Vogel 2009, S. 70 ff.). Das bedeutet, dass auch heute noch in den meisten Fällen die Frau in den Hof einheiratet und nicht umgekehrt. Gemäß den in Österreich bestehenden Traditionen übernimmt das junge Bauern-Ehepaar mit dem Zeitpunkt der Heirat die Leitung des Hofs. Dennoch ist die Beziehung zwischen den Generationen stark davon geprägt, wer das Sagen am Hof hat. Durch die vorwiegend patrilineare Vererbung stehen sich im Generationenverhältnis meist Vater und Sohn auf der einen, Schwiegermutter und 
eingeheiratete Schwiegertochter auf der anderen Seite gegenüber. Besonders häufig scheinen dabei - wie die von uns geführten Interviews zeigen - nach wie vor Konflikte zwischen der Hof-fremden, angeheirateten Schwiegertochter und deren Schwiegermutter zu sein.

Solche Konflikte zwischen der angeheirateten Schwiegertochter und den Schwiegereltern, insbesondere der Schwiegermutter, haben im bäuerlichen Milieu eine lange Tradition (z.B. Goldbrunner 2007). Auch wenn sich die Altbauern wünschen, dass der männliche Hofnachfolger eine Frau findet, die ihn bei der Hof- und Hausarbeit unterstützt und mit der er eine Familie gründen kann, kommen die Schwiegermütter mit dem neuen Familienmitglied und den damit verbundenen Veränderungen häufig schwer zurecht. Ein gewisses Maß an Offenheit für derartige Veränderungen erweist sich jedoch für den Fortbestand des Hofes als notwendig, insoweit sich die Schwiegertöchter heute nicht mehr in vergleichbarer Weise wie früher den bestehenden Verhältnissen unterordnen. Besonders schwerwiegend geraten die Konflikte tendenziell dann, wenn sich die Schwiegertochter aktiv gegen veraltete Geschlechterrollen auflehnt und sich nicht an die Traditionen und alltäglichen Praxen des Hofes anpasst (Goldberg 2003). Das einst typischerweise hierarchische Verhältnis zwischen der Schwiegermutter als Lehrerin und der Schwiegertochter als Schülerin, die erlernt, wie der Haushalt zu führen ist und die Hofarbeiten zu erledigen sind, wird zunehmend unwahrscheinlich, womit ein Machtverlust der Schwiegermütter einhergeht. Gleichzeitig werden die Schwiegermütter bei der Beaufsichtigung der Kinder und den Haushalts- und Hofarbeiten benötigt. Außerdem verbringen aufgrund der geschlechtsspezifischen Arbeitsteilung Schwiegermütter und -töchter bei den Arbeiten im Haushalt und den Hofarbeiten zumeist viel Zeit miteinander, was das Konfliktpotenzial erhöht.

Abgrenzungsprobleme gegenüber der Schwiegermutter schildern beispielsweise Andreas Sch. (51) und seine Gattin Elfriede (49). Unter vorgeschobenen Argumenten versuche die Schwiegermutter alles, was im Haus und am Hof geschieht, zu kontrollieren und verletze dabei nicht nur die Privatsphäre des Sohnes und der Schwiegertochter, sondern auch jene der Enkelkinder. Der Kontrollzwang der Schwiegermutter ginge so weit, dass sich das Ehepaar gezwungen sieht, das Auto zu nutzen, um private Dinge zu besprechen. Aus Sicht des Sohnes leidet insbesondere seine Frau unter seiner Mutter, zumal ihre Arbeitsleistung stets von der Schwiegermutter bemängelt wird.

„Wie sie [die Schwiegermutter] vorhin reingekommen ist, ist sie nur gekommen, weil die Frau fortgefahren ist. Weil das ist der Kontrollgang. Weil dann geht sie in die Küche rein, schauen. Dann geht sie in den Kühlraum rein, schauen. Und wenn irgendwas nicht passt, sobald die Elfriede heimkommt, sagt sie ihr: ,Schau her, das und das passt nicht ' [...] Oder zu den Buben ins Zimmer, ich meine, da geh' ich auch nicht rein - das gehört sich einfach nicht! [...] Aber sie macht das immer unter einem gewissen Vorwand. Sie ist ganz schlau damit auch, so wie sie vorhin gekommen ist, dass sie Geld wechseln will. Damit es nicht auffällt, was sie in Wahrheit heroben tun will! (lacht) Ich meine, für mich ist das ja nicht so schlimm, aber für die Elfriede [...] für sie ist es schon hart!“ (Andreas Sch., 51) 
Elfriede Sch. versucht die Forderungen und die Einmischung der Schwiegermutter weitestgehend zu ignorieren und hat sich von den Erwartungen der Schwiegermutter und einem als hierarchisch verstandenen Generationenverhältnis inzwischen weitgehend losgelöst. Sie sieht sich ihrem Mann gegenüber als gleichberechtigt an und bricht damit die tief verankerten und über Jahrhunderte tradierten Rollenvorstellungen über das Geschlechterverhältnis im bäuerlichen Milieu auf, was unweigerlich zu Konflikten führt. In diesen Konfliktsituationen wird von männlichen Hoferben wie Andreas Sch. oftmals verlangt, zu den Erwartungen der Ehefrau wie auch zu denen der Mutter Stellung zu beziehen, was sie als unangenehmen Loyalitätskonflikt erleben. Gestaltet sich das Verhältnis zwischen den Altbauern und den Jungbauern und speziell zwischen Schwiegermutter und Schwiegertochter konfliktreich, wird insbesondere die Frage der Pflege umso schwieriger zu verhandeln. Elfriede Sch. berichtet, dass sie die Pflege der Schwiegermutter keinesfalls alleine übernehmen möchte. Sie und ihr Mann haben letztlich gemeinsam entschieden, dafür externe Hilfe in Anspruch zu nehmen.

Durch den strukturellen Wandel in der Landwirtschaft und den Wandel der Geschlechterrollen hat sich die Situation der Jungbäuerinnen am Hof zum Teil erheblich verändert. Auf Nebenerwerbsbetrieben geht meist der Mann einer außerlandwirtschaftlichen Erwerbstätigkeit nach, was eigenständige Entscheidungen der eingeheirateten Jungbäuerin erforderlich macht und ihr eine stärkere Position verleiht. Zum Teil kommen Jungbäuerinnen auch aus anderen Milieus, wo in der Regel eine geringere Bereitschaft vorherrscht, traditionelle Geschlechterrollen zu übernehmen. Dabei scheinen Frauen einerseits, wie u.a. Hildenbrand zeigte, die „Agentinnen der Modernisierung“ auf den Bauernhöfen zu sein, andererseits geht jedoch der Autonomiegewinn von Frauen im Zuge gewandelter Rollenerwartungen auch mit gesteigerten Herausforderungen für diese einher (vgl. Hildenbrand 2005, S. 124).

Die 38-jährige Martina R. ist ein Beispiel für eine hoch gebildete Jungbäuerin, die nicht aus dem bäuerlichen Milieu stammt und den Bergbauernbetrieb im Nebenerwerb bewirtschaftet, während ihr Mann als Handwerker tätig ist. Trotz des Widerstandes der Schwiegermutter stellte Martina R. den Betrieb völlig um und probiert nun neue Produktionsweisen, neue Tierhaltungsformen und neue Vermarktungsstrategien aus.

„Ja und dann bin ich halt HAK [zur Handelsakademie, Anm. d.Verf.] gegangen und habe Matura gemacht [...]. Ich habe dann gleich einmal bei uns daheim einen Job gehabt, also ich habe [...] bei den Bauerndörfern in der Rezeption arbeiten können. [...] ich bin auch immer willig, dass ich etwas Neues dazulerne oder so. [...] Ich bin da nicht so festgefahren, weil da hat es zwischen schon einmal, eine Serie Würstel gegeben, die war nicht zum Essen. Dann habe ich gesagt zur Oma: ,Du bitte, ich kann das nicht nach Gefühl! Ich brauche da ein Rezept!' Und dann gehe ich her und ich wiege das ab und dann sind die Würstel immer gleich. Und mittlerweile machen wir das auch so und jetzt werden sie immer gleich. Aber wir, ich tu auch gern einmal was anderes ausprobieren.“ (Martina R., 38).

Konflikte zwischen den Generationen betreffen häufig auch die Beziehung zwischen Vater und Sohn, d.h. zwischen dem ehemaligen Betriebsleiter und dem Hof- 
nachfolger, die zumeist bereits vor der Hofübernahme eng miteinander arbeiteten. Während es für den Altbauern in der Regel noch ein Privileg darstellte, den Hof des Vaters zu übernehmen, ist es heute keineswegs mehr selbstverständlich, dass der Betrieb überhaupt weitergeführt wird. Hinzu kommt, dass sich die Halbwertszeit des Wissens der älteren Generation (Brake und Büchner 2007, S. 204) gerade in der Landwirtschaft enorm verringert hat - betriebliche Innovationen sind von großer Bedeutung für das langfristige Überleben der Höfe. Diese beiden Veränderungen tragen zu einem Machtverlust der älteren Generation bei und können das gegenseitige Verständnis von Vater und Sohn erschweren.

Der 39-jährige Michael F. berichtet über Konflikte mit dem Vater, die aus seiner Sicht zwar lediglich Kleinigkeiten betreffen und nach einer kurzen Eskalation auch wieder ein Ende finden, die er aber als durchaus „,nervtötend“ bezeichnet. Er beschreibt den Vater-Sohn-Konflikt selbst als einen Generationenkonflikt, der zudem durch den Altersstarrsinn seines Vaters verstärkt werde - von dem er fürchtet, dass auch er selbst ihn im Alter zeigen könnte.

„Ahm, aber den Generationskonflikt, den gibt es bei uns genauso. Und wahrscheinlich auch viel, viel heftiger, weil ich genauso ein sturer Mensch oder Mann sein kann wie mein Vater. Also bei uns fliegen regelrecht die Fetzen. Also verbal jetzt einmal. Und das kann zehn Minuten dauern und die nächsten zehn Minuten wird wieder ganz normal gearbeitet und die nächsten drei Stunden. [D]a geht es auch meistens, [...] um Kleinigkeiten. Weiß' nicht, ob es Kleinigkeiten sind, aber nicht jetzt um irgendwelche Katastrophen. Was sicher ein großes Problem ist [...], ist, das Altwerden. Das ist ein Thema, das in der Landwirtschaft sicher ein größeres Problem darstellt, weil man ja den ganzen Tag mit [...] allen Leuten auf einem Betrieb ist und auch arbeitet. Und das Alter bringt eben Sachen mit sich, die man nicht wirklich glauben will. Auch selber nicht. Man glaubt immer, die, ah, Alten werden schwieriger oder was, aber das ist einfach so, wir werden es wahrscheinlich auch irgendwann. Nur, es ist einfach nervtötend. Und mit dem umzugehen, ist relativ schwierig." (Michael F., 39)

Ähnliche Spannungen kann es auch geben, wenn die Tochter den Hof übernimmt oder selbst wenn die Erbfolge matrilinear verläuft, also der Hof von der Mutter an die Tochter vererbt wird. So berichtet beispielsweise die 54-jährige Aloisia, die gerne gefragt worden wäre, ob sie den Hof überhaupt übernehmen wolle, über die Dominanz ihrer Mutter: „Weil, meine Mama war eine sehr dominante, da hat es einfach ihren Weg gegeben und da hast du müssen einfach diesen Weg mitgehen.“

Die Situation der eingeheirateten Schwiegersöhne unterscheidet sich jedoch maßgeblich von jener der angeheirateten Schwiegertöchter, da ihre Arbeitskraft auf den Höfen aus der Sicht der Elterngeneration stärker gebraucht wird und ihnen in der Regel mehr Mitspracherecht eingeräumt wird. Darüber hinaus gehen die eingeheirateten Schwiegersöhne häufig einer außerlandwirtschaftlichen Erwerbstätigkeit nach und sind dadurch weniger am Hof. 


\section{Zusammenfassung und Diskussion}

Im vorliegenden Beitrag wurde untersucht, inwiefern die Generationenbeziehungen im bäuerlichen Milieu am Beginn des 21. Jahrhunderts durch Persistenz und Wandel zugleich charakterisiert sind. Einerseits sind sie von einer starken Kontinuität der Familiensolidarität geprägt. Für die Altbauern ist es selbstverständlich, bei der Arbeit am Hof und bei der Betreuung der Enkel mitzuhelfen, solange sie dazu in der Lage sind. Im Gegenzug besteht auch bei den Jungbauern eine hohe Bereitschaft, die Altbauern mitzuversorgen und die Pflege so zu organisieren, dass diese möglichst lange am Hof bleiben können. Diese wechselseitige Unterstützung wird von den Betroffenen nicht nur als funktionale Tauschbeziehung gesehen, das Zusammenleben am Hof und die emotionale Verbundenheit zwischen den drei Generationen wird vielfach als sehr beglückend und als erstrebenswerte Lebensform empfunden.

Andererseits lassen sich nicht nur vielfältige Kontinuitäten, sondern lässt sich auch ein bedeutender Wandel der Solidaritäts- und Konfliktmuster beobachten. Den Kern dieses Wandels bilden Enthierarchisierungs- und Individualisierungsprozesse, die mit Machtverschiebungen zwischen den Generationen einhergehen und von einer Emotionalisierung der Beziehungen begleitet werden. Selbstbestimmtheit, Mitsprache und gemeinsame Aushandlung bestimmen zunehmend das soziale Klima auf den österreichischen Bauernhöfen des 21. Jahrhunderts. Die besondere Brisanz dieser Entwicklungen erschließt sich gleichwohl erst, wenn man die historischen Ausgangsbedingungen und insbesondere die Geschlechterrollen im bäuerlichen Milieu mitbedenkt. Vor diesem Hintergrund erweist sich (1.) ein Wandel von der verpflichtenden zur freiwilligen Generationensolidarität, (2.) von traditionellen Abhängigkeitsverhältnissen zu einer autonomeren Lebensführung sowie mehr Privatsphäre und (3.) vom patriarchalen Gehorsam zur egalitären Familienkommunikation als kennzeichnend für das bäuerliche Milieu.

Dabei ist der von uns beobachtete Wandel der Generationenbeziehungen alles andere als spannungsfrei. Zwar hat die Freiwilligkeit intergenerationaler Interaktionen zweifelsfrei zugenommen, erfährt die jüngere Generation heute mehr Autonomie und führt zusehends Aushandlungsprozesse auf Augenhöhe mit der Elterngeneration; doch das „Spannungsfeld zwischen Unterstützung und Einmischung, Engagement und vornehmer Zurückhaltung" (Brake und Büchner 2007, S. 200) ist durch die räumliche, emotionale und betrieblich notwendige Nähe im bäuerlichen Milieu dennoch ausgeprägter als außerhalb der Landwirtschaft.

Ambivalenzen in den Generationenbeziehungen ergeben sich vor allem, wo die Altbauern nach Bewahrung streben, während die Jungbauern und -bäuerinnen Innovationen vorantreiben (müssen). Im Ergebnis erweisen sich die von uns analysierten Familien als Mischtypen zwischen Solidarität (übereinstimmend bewahren) und Emanzipation (einvernehmlich entwickeln). ${ }^{9}$ Auf das bäuerliche Milieu scheint

\footnotetext{
9 Der Typus „Kaptivation“ findet sich in den gegenwärtigen Familienkonstellationen unserer Befragten nicht, taucht jedoch vereinzelt in Erzählungen zur Hofgeschichte und in den Kindheitserinnerungen insbesondere der älteren Befragten auf. Das Aufweisen des Typus „Atomisierung“ in unserer Stichprobe muss von vornherein als unwahrscheinlich gelten, da wir nur Bäuerinnen und Bauern auf noch existierenden Landwirtschaften und keine Männer und Frauen, die die Landwirtschaft aufgegeben hatten, interviewt haben.
} 
insofern die Formel „Modernising to remain traditional“ (Hennon und Hildenbrand 2005) zuzutreffen.

Fernab dieser generellen Muster lassen sich auch Abweichungen in den Erzählungen der Bauern und Bäuerinnen erkennen: Während es an manchen Höfen bereits früh schon relativ egalitäre Generationenbeziehungen gab (in einigen Fällen auch nur zu einem Elternteil und nicht zu Vater und Mutter) und auch Frauen viel Mitspracherecht hatten, schreitet die Enthierarchisierung der Beziehungen zwischen den Generationen und Geschlechtern an anderen Höfen bestenfalls langsam voran. Diese Ungleichzeitigkeit der Entwicklung lässt sich auf verschiedene soziale, betriebsbiographische, ökonomische und geographische Faktoren zurückführen, wie etwa den Zeitpunkt der Heirat und Betriebsübernahme, das Alter der Kinder, die Pflegebedürftigkeit und das Ableben von Personen aus der ältesten am Hof lebenden Generation, die ökonomischen Rahmenbedingungen, den Bildungsgrad der Hofnachfolge, die Produktionsbranche oder die Nähe zum städtischen Milieu. In zukünftigen Forschungsarbeiten gilt es, diese Ungleichzeitigkeiten vermehrt in den Blick zu nehmen, um ein noch differenzierteres Verständnis der Generationenbeziehungen im bäuerlichen Milieu zu erlangen. Das Anliegen des vorliegenden Beitrags lag dagegen nicht in der Typologisierung von Intergenerationenbeziehungen, sondern in deren Historisierung und der Rekonstruktion intergenerationaler Solidaritätsund Konfliktmuster unter der Einbeziehung von Geschlechteraspekten.

Abschließend lässt sich festhalten, dass es auf den österreichischen Bauernhöfen in den letzten Jahrzehnten zu einem tiefgreifenden Wandel der Generationenbeziehungen und Geschlechterrollen gekommen ist. Er lässt erkennen, dass auch die bäuerlichen Lebensverhältnisse von umfassenden gesellschaftlichen Veränderungsprozessen erfasst werden. Entgegen gängiger Individualisierungstheorien lassen sich bäuerliche Traditionen jedoch durchaus mit diesen Entwicklungen vereinbaren. Grundvoraussetzung dafür ist die kommunikative Aufrechterhaltung und Pflege der Solidaritätsbeziehungen zwischen den Familienmitgliedern, auf deren Grundlage sich Arbeits-, Freizeit- und Familienarrangements erfolgreich aushandeln und alte und neue Generationenkonflikte erfolgreich bewältigen lassen.

Funding Open access funding provided by University of Graz.

Open Access Dieser Artikel wird unter der Creative Commons Namensnennung 4.0 International Lizenz veröffentlicht, welche die Nutzung, Vervielfältigung, Bearbeitung, Verbreitung und Wiedergabe in jeglichem Medium und Format erlaubt, sofern Sie den/die ursprünglichen Autor(en) und die Quelle ordnungsgemäß nennen, einen Link zur Creative Commons Lizenz beifügen und angeben, ob Änderungen vorgenommen wurden.

Die in diesem Artikel enthaltenen Bilder und sonstiges Drittmaterial unterliegen ebenfalls der genannten Creative Commons Lizenz, sofern sich aus der Abbildungslegende nichts anderes ergibt. Sofern das betreffende Material nicht unter der genannten Creative Commons Lizenz steht und die betreffende Handlung nicht nach gesetzlichen Vorschriften erlaubt ist, ist für die oben aufgeführten Weiterverwendungen des Materials die Einwilligung des jeweiligen Rechteinhabers einzuholen.

Weitere Details zur Lizenz entnehmen Sie bitte der Lizenzinformation auf http://creativecommons.org/ licenses/by/4.0/deed.de. 


\section{Literatur}

Bauman, Z. (1992). Moderne und Ambivalenz. Das Ende der Eindeutigkeit. Hamburg: Junius.

Beck, U., \& Beck-Gernsheim, E. (1990). Das ganz normale Chaos der Liebe. Frankfurt a. M.: Suhrkamp.

Beck, U., \& Beck-Gernsheim, E. (1994). Individualisierung in modernen Gesellschaften - Perspektiven und Kontroversen einer subjektorientierten Soziologie. In U. Beck \& E. Beck-Gernsheim (Hrsg.), Riskante Freiheiten. Individualisierung in modernen Gesellschaften (S. 10-39). Frankfurt a. M.: Suhrkamp.

Beck-Gernsheim, E. (1997). Stabilität der Familie oder Stabilität des Wandels? Zur Dynamik der Familienentwicklung. In U. Beck (Hrsg.), Individualisierung und Integration. Neue Konfliktlinien und neuer Integrationsmodus (S. 65-80). Opladen: Leske \& Budrich.

Beham-Rabanser, M., Berghammer, C., \& Zartler, U. (2019). Partnerbeziehungen zwischen Flexibilität und Stabilität. In J. Bacher, A. Grausgruber, M. Haller, F. Höllinger, D. Prantner \& R. Verwiebe (Hrsg.), Sozialstruktur und Wertewandel in Österreich. Trends 1986-2016 (S. 179-210). Wiesbaden: Springer VS.

Bengtson, V. L. (2001). The Burgess Award lecture: Beyond the nuclear family: The increasing importance of multigenerational bonds. Journal of Marriage and Family, 63(1), 1-16.

Bengtson, V.L., \& Kuypers, J.A. (1971). Generational difference and the development stake. Aging and human development, 2(4), 249-260.

Bengtson, V. L., \& Roberts, R. (1991). Intergenerational solidarity in ageing families: An example of formal theory construction. Journal of Marriage and Family, 53(4), 856-870.

Bengtson, V. L., Giarrusso, R., Mabry, B. J., \& Silverstein, M. (2002). Solidarity, conflict, and ambivalence: Complementary or competing perspectives on intergenerational relationships? Journal of Marriage and Family, 64(3), 568-576.

Bertram, H. (2000). Die verborgenen familiären Beziehungen in Deutschland: Die multilokale Mehrgenerationenfamilie. In M. Kohli \& M. Szydlik (Hrsg.), Generationen in Familie und Gesellschaft (S. 97-121). Opladen: Leske + Budrich.

Brake, A., \& Büchner, P. (2007). Großeltern in Familien. In J. Ecarius (Hrsg.), Handbuch Familie (S. 199-219). Wiesbaden: VS Verlag für Sozialwissenschaften.

Brauer, K. (2002). Ein Blick zurück nach vorn. Generationenbeziehungen im Stadt-Land-Vergleich. In G. Burkart \& J. Wolf (Hrsg.), Lebenszeiten. Erkundungen zur Soziologie der Generationen (S. 175-195). Wiesbaden: Springer.

Bruckmüller, E. (2002). Vom „Bauernstand“ zur „Gesellschaft des ländlichen Raums“ - Sozialer Wandel in der bäuerlichen Gesellschaft des 20. Jahrhunderts. In E. Bruckmüller, R. Hanisch \& R. Sandgruber (Hrsg.), Geschichte der österreichischen Land- und Forstwirtschaft im 20. Jahrhundert. Band 1: Politik - Gesellschaft - Wirtschaft (S. 409-592). Wien: Ueberreuter.

Bundesministerium für Land- und Forstwirtschaft, Umwelt und Wasserwirtschaft (Hrsg.). (2014). Grüner Bericht 2014. Bericht über die Situation der österreichischen Land- und Forstwirtschaft. https:// gruenerbericht.at/cm4/jdownload/send/2-gr-bericht-terreich/1392-gb2014. Zugegriffen: Feb. 2020.

Bundesminister für Land- und Fortwirtschaft (Hrsg.). (2016). Grüner Bericht 2016. Bericht über die Situation der österreichischen Land- und Forstwirtschaft. Wien. https://gruenerbericht.at/cm4/jdownload/ send/2-gr-bericht-terreich/1650-gb2016 Zugegriffen: Feb. 2020.

Bundesministerium für Nachhaltigkeit und Tourismus (Hrsg.). (2019). Grüner Bericht 2019. Die Situation der österreichischen Land- und Forstwirtschaft. Wien. https://gruenerbericht.at/cm4/jdownload/send/ 2-gr-bericht-terreich/2007-gb2019. Zugegriffen: Feb. 2020.

Burkart, G. (2008). Familiensoziologie. Konstanz: UVK.

Clarke, E. J., Preston, M., Raksin, J., \& Bengtson, V.L. (1999). Types of conflicts and tensions between older parents and adult children. The Gerontologist, 39(3), 261-270.

Cooney, T. M., \& Dykstra, P.A. (2013). Theories and their empirical support in the study of intergenerational family relationships in adulthood. In M.A. Fine and F.D. Finchman (Hrsg.), Handbook of family theories: A content-based approach (S. 356-378). New York: Routledge.

Eder, A., \& Haring, S. A. (2017). Familienleben und Generationenbeziehungen. In F. Höllinger, A. Eder, E.-M. Griesbacher \& S. A. Haring, Bäuerliche Lebenswelten in Österreich am Beginn des 21. Jahrhunderts (S. 125-168). Graz: Leykam Verlag.

Fliege, T. (1998). Bauernfamilien zwischen Tradition und Moderne. Eine Ethnographie bäuerlicher Lebensstile. Frankfurt a. M.: Campus.

Geserick, C., Kapelle, O., \& Kaindl, M. (2006). Situation der Bäuerinnen in Österreich 2006. Working Paper 68. Wien: Institut für Familienforschung der Universität Wien. 
Goldberg, C. (2003). Postmoderne Frauen in traditionalen Welten: zur Weiblichkeitskonstruktion von Bäuerinnen. Frankfurt a. M.: Peter Lang.

Goldbrunner, H. (2007). Die Beziehungen zwischen den Generationen im Spannungsfeld von Unternehmen und Familie. In Bundesarbeitsgemeinschaft der Landwirtschaftlichen Familienberatungen und Sorgentelefone e. V. (BAG) (Hrsg.), Generationskonflikte auf landwirtschaftlichen Betrieben - ihre Bedeutung und Bearbeitung aus systemischer Sicht. Dokumentation einer internationalen Tagung im Christian-Jensen-Kolleg Breklum vom 26. bis 29. Juni 2006. Schwalmstadt: BAG.

Groier, M. (2004). Wachsen und Weichen. Rahmenbedingungen, Motivationen und Konsequenzen von Betriebsaufgaben in der österreichischen Landwirtschaft. Forschungsbericht Nr. 51 der Bundesanstalt für Bergbauernfragen. Wien: Bundesanstalt für Bergbauernfragen.

Helfferich, C. (2017). Familie und Geschlecht: eine neue Grundlegung der Familiensoziologie. Opladen: Barbara Budrich.

Hennon, C., \& Hildenbrand, B. (2005). Modernizing to remain traditional. Farm families maintaining a valued lifestyle. Journal of Comparative Family Studies, 36(3), 505-520.

Hermann, H. (2008). Liebesbeziehungen - Lebensentwürfe. Eine Soziologie der Partnerschaft. 4. Aufl. Münster: Telos.

Hildenbrand, B. (2005). Landfamilien und Bauernfamilien. In S. Beetz, K. Brauer \& C. Neu (Hrsg.), Handwörterbuch zur ländlichen Gesellschaft in Deutschland (S. 121-127). Wiesbaden: VS Verlag für Sozialwissenschaften.

Hildenbrand, B., Bohler, K. F., Walter, J., \& Schmitt, R. (1992). Bauernfamilien im Modernisierungsprozeß. Frankfurt a. M.: Campus.

Hoffmann-Nowotny, H.-J. (1995). Die Gesellschaft auf dem Weg zur individualisierten Selbstauflösung? In P. Meinrad, J.-L. Lambert, C. Ermert \& B. Plancherel (Hrsg.), Familie im Wandel. Bern: Universitätsverlag Freiburg Schweiz.

Höllinger, F., Eder, A., Griesbacher, E.-M., \& Haring, S. A. (2017). Bäuerliche Lebenswelten in Österreich am Beginn des 21. Jahrhunderts. Graz: Leykam Verlag.

Homans, G. C. (1961). Social behavior: Its elementary forms. New York: Harcourt.

Höpflinger, F. (1999). Generationenfrage-Konzepte, theoretische Ansätze und Beobachtungen zu Generationenbeziehungen in späteren Lebensphasen. Lausanne: Réalités sociales.

Kaindl, M., \& Schipfer, R. K. (2019). Familie in Zahlen 2019. Statistische Informationen zu Familien in Österreich. Österreichisches Institut für Familienforschung an der Universität Wien. https://doi.org/ 10.25365/phaidra.117. Zugegriffen: Feb. 2020.

Kalkus, A. (2016). Der Übergabevertrag. In: Österreichische Landjugend (Hrsg.), Hofübergabe/Hofübernahme (S. 33-38). http://jungbauern.at/wp-content/uploads/2016/05/LJ-Hofuebergabe-Broschuere_ 160216ok_72dpi_2_.pdf. Zugegriffen: Feb. 2020.

Kaplan, M. S., Nussbaum, J. F., Becker, J. C., \& Fowler, C. (2009). Communication barriers to family farm succession planning. Journal of Extension, 47(5), 1-9.

Kirner, L. (2017). Wirtschaftliche Entwicklung der bäuerlichen Familienbetriebe in Österreich unter Berücksichtigung internationaler und nationaler Trends. In F. Höllinger, A. Eder, E.-M. Griesbacher \& S. A. Haring, Bäuerliche Lebenswelten in Österreich am Beginn des 21. Jahrhunderts (S. 41-66). Graz: Leykam.

Kohli, M., \& Szydlik, M. (2000). Generationen in Familie und Gesellschaft. Opladen: Leske + Budrich.

Kopp, J., \& Steinbach, A. (2009). Generationenbeziehungen. Kölner Zeitschrift für Soziologie und Sozialpsychologie, 61(2), 283-294.

Kuckartz, U. (2012). Qualitative Inhaltsanalyse: Methoden, Praxis, Computerunterstützung. Juventa: Beltz.

Langthaler, E. (2012). Balancing between autonomy and dependence. Family farming and agrarian change in lower Austria, 1945-1980. In G. Bischof, F. Plasser \& E. Maltschnig (Hrsg.), Austrian Lives (S. 383-404). New Orleans: New Orleans University Press.

Larcher, M., \& Vogel, S. (2009). Geschlechterspezifische Unterschiede im Hofübergabeprozess in Österreich. In T. Oedl-Wieser \& I. Darnhofer (Hrsg.), Jahrbuch der Gesellschaft der österreichischen Agrarökonomie, 18(2), 67-78.

Lüscher, K. (1993). Generationenbeziehungen - Neue Zugänge zu einem alten Thema. In K. Lüscher \& F. Schultheis (Hrsg.), Generationenbeziehungen in „postmodernen“ Gesellschaften (S. 17-50). Konstanz: UVK.

Lüscher, K. (2000). Die Ambivalenz von Generationenbeziehungen - eine allgemeine heuristische Hypothese. In M. Kohli \& M. Szydlik (Hrsg.), Generation in Familie und Gesellschaft. (S. 139-161). Wiesbaden: VS Verlag für Sozialwissenschaften. 
Lüscher, K., Hoff, A., Lamura, G., Renzi, M., Sánchez, M., Viry, G., Widmer, E., Klimczuk, A., \& de Salles Oliveira, P. (2014). Generationen, Generationenbeziehungen, Generationenpolitik. Ein mehrsprachiges Kompendium. Konstanz: UVK.

Marbach, J. H. (1994). Tauschbeziehungen zwischen Generationen: Kommunikation, Dienstleistungen und finanzielle Unterstützung in Dreigenerationenfamilien. In W. Biehn (Hrsg.), Eigeninteresse oder Solidarität. Beziehungen in modernen Mehrgenerationenfamilien (S. 163-196). Wiesbaden: VS Verlag für Sozialwissenschaften.

Marshall, V. W., Matthews, S. H., \& Rosenthal C. H. (1993). Elusiveness of family life: A challenge for the sociology of ageing. Annual Review of Geriatrics, 13, 39-72.

Meyer, T. (2008). Private Lebensformen im Wandel. In R. Geißler (Hrsg.), Die Sozialstruktur Deutschlands (S. 331-357). Wiesbaden: Springer.

Mitterauer, M., \& Sieder, R. (1991). Vom Patriarchat zur Partnerschaft. Zum Strukturwandel der Familie. München: C.H. Beck.

Peuckert, R. (2008). Familienformen im sozialen Wandel. Wiesbaden: VS Verlag für Sozialwissenschaften.

Pillemer, K., \& Müller-Johnsin, K. (2007). Generationenambivalenzen. Ein neuer Zugang zur Erforschung familialer Generationenbeziehungen. In F. Lettke \& A. Lange (Hrsg.), Generationen und Familien (S. 130-157). Frankfurt a. M.: Suhrkamp.

Pongratz, H. (1990). Landwirtschaft und Gesellschaft. Zum Wandel des gesellschaftlichen Umfelds der bäuerlichen Familien. In Bildungsstätte des Bayerischen Bauernverbands (Hrsg.), Herrschinger Hefte. Bd. 10 (S. 18-32). Herrsching: Bildungsstätte des Bayrischen Bauernverbands.

Pongratz, H. (1991). Bäuerliche Tradition im sozialen Wandel. Kölner Zeitschrift für Soziologie und Sozialpsychologie, 43(1), 235-247.

Quendler, E., Brückler, M., \& Resl, T. (2015). Außerfamiliäre Hofübergabe in Österreich. Bedarfsstudie für eine Informations- und Bildungsoffensive basierend auf österreichweiten Befragungen von Landwirtinnen. Wien: Bundesanstalt für Agrarwirtschaft. https://landjugend.at/fileadmin/user_upload/ Bund/Programm/LW_und_Umwelt/Hofuebergabe/Ausserfamiliaere_Hofuebergabe/Bedarfsstudie_ Ausserfamiliaere_Hofuebergabe_AWI_Juni_2015_ohne_Anhang.pdf. Zugegriffen: Feb. 2020.

Rosenbaum, H. (1982). Formen der Familie. Untersuchungen zum Zusammenhang von Familienverhältnissen, Sozialstruktur und sozialem Wandel in der deutschen Gesellschaft des 19. Jahrhunderts. Frankfurt a. M.: Suhrkamp.

Rosenbaum, H. (2011). Zwischen Konflikt und Solidarität. In R. Mohrmann (Hrsg.), Generationenbeziehungen in Familie und Gesellschaft (S. 11-34). Münster: Waxmann Verlag.

Rossier, R. (2005). Role models and farm development options: A comparison of seven Swiss farm families. Journal of Comparative Family Studies, 36(3), 399-417.

Stiglbauer, A., \& Weiss, C. R. (2000). Family and non-family succession in the Upper-Austrian farm sector. Cahiers d'Économie et Sociologie Rurales, 54, 3-26.

Szydlik, M. (2000). Lebenslange Solidarität? Generationenbeziehungen zwischen erwachsenen Kindern und Eltern. Opladen: Leske + Budrich.

Szydlik, M. (2008). Intergenerational solidarity and conflict. Journal of Comparative Family Studies, 39(1), 97-114.

Tartler, R. (1961). Das Alter in der modernen Gesellschaft. Stuttgart: Enke.

Westermayer, T. (2006). Die Ich-AG im Walde. Arbeit in ländlichen Räumen der postindustriellen Gesellschaft am Beispiel forstlicher Dienstleistungsunternehmen. Berliner Journal für Soziologie, 16, 211-225.

Anja Eder geb. 1984. Dr. phil., seit 2017 Postdoktorandin am Institut für Soziologie und in der Koordinationsstelle für Geschlechterstudien und Gleichstellung an der Universität Graz. Forschungsschwerpunkte: Soziale Ungleichheitsforschung, politische Soziologie, international vergleichende Gesellschaftsanalyse, angewandte Sozialforschung. Ausgewählte Veröffentlichungen: (mit S. A. Haring) Familienleben und Generationenbeziehungen, in: F. Höllinger, A. Eder, E.-M. Griesbacher \& S. A. Haring (Hrsg.), Bäuerliche Lebenswelten in Österreich am Beginn des 21. Jahrhunderts, 2017; (mit M. Hadler und M. Schweighart) „Komfortzone“ Mittelschicht? Eine quantitative Analyse der subjektiven sozialen Position der Österreicher/-innen 1993-2016, in: N. Burzan (Hrsg.), Komplexe Dynamiken globaler und lokaler Entwicklungen. Verhandlungen des 39. Kongresses der Deutschen Gesellschaft für Soziologie in Göttingen 2018, 2019. 
Sabine A. Haring-Mosbacher geb. 1970. Dr. phil., Assoz. Prof. am Institut für Soziologie der Universität Graz, Lektorin an der Universität Innsbruck. Forschungsschwerpunkte: Historische und Politische Soziologie, zentraleuropäische Geschichte des 19. und 20. Jahrhunderts, Emotionssoziologie, Soziologie der Gewalt. Ausgewählte Veröffentlichungen: Verheißung und Erlösung. Religion und ihre weltlichen Ersatzbildungen in Politik und Wissenschaft, 2008; (mit Helmut Kuzmics) Emotion, Habitus und Erster Weltkrieg. Soziologische Studien zum militärischen Untergang der Habsburger Monarchie, 2013.

Franz Höllinger geb. 1957. Dr. phil., seit 1996 a. o. Universitätsprofessor am Institut für Soziologie an der Universität Graz. Forschungsschwerpunkte: interkulturell vergleichende Sozialforschung, Religionssoziologie, Familiensoziologie. Ausgewählte Veröffentlichungen: (mit A. Eder, E.-M. Griesbacher und S. A. Haring) Bäuerliche Lebenswelten in Österreich am Beginn des 21. Jahrhunderts, 2017; (mit M. Haller, J. Bacher, A. Grausgruber, D. Prandner und R. Verwiebe) Sozialstruktur und Wertewandel in Österreich. Trends 1986-2016, 2019. 